\title{
Snake in the Clouds: A new nearby dwarf galaxy in the Magellanic bridge*
}

\author{
Sergey E. Koposov, ${ }^{1,2} \dagger$ Matthew G. Walker, ${ }^{1}$ Vasily Belokurov, ${ }^{2,3}$ Andrew R. Casey, ${ }^{4,5}$ \\ Alex Geringer-Sameth, ${ }^{6}$ Dougal Mackey, ${ }^{7}$ Gary Da Costa, ${ }^{7}$ Denis Erkal ${ }^{8}$, \\ Prashin Jethwa ${ }^{9}$, Mario Mateo, ${ }^{10}$, Edward W. Olszewski ${ }^{11}$ and John I. Bailey III ${ }^{12}$ \\ ${ }^{1}$ McWilliams Center for Cosmology, Carnegie Mellon University, 5000 Forbes Ave, 15213, USA \\ ${ }^{2}$ Institute of Astronomy, University of Cambridge, Madingley road, CB3 OHA, UK \\ ${ }^{3}$ Center for Computational Astrophysics, Flatiron Institute, 162 5th Avenue, New York, NY 10010, USA \\ ${ }^{4}$ School of Physics and Astronomy, Monash University, Clayton 3800, Victoria, Australia \\ ${ }^{5}$ Faculty of Information Technology, Monash University, Clayton 3800, Victoria, Australia \\ ${ }^{6}$ Astrophysics Group, Physics Department, Imperial College London, Prince Consort Rd, London SW7 2AZ, UK \\ ${ }^{7}$ Research School of Astronomy and Astrophysics, Australian National University, Canberra, ACT 2611, Australia \\ ${ }^{8}$ Department of Physics, University of Surrey, Guildford, GU2 7XH, UK \\ ${ }^{9}$ European Southern Observatory, Karl-Schwarzschild-Str. 2, 85748 Garching, Germany \\ ${ }^{10}$ Department of Astronomy, University of Michigan, 311 West Hall, 1085 S University Avenue, Ann Arbor, MI 48109, USA \\ ${ }^{11}$ Steward Observatory, The University of Arizona, 933 N. Cherry Avenue., Tucson, AZ 85721, USA \\ ${ }^{12}$ Leiden Observatory, Leiden University, Niels Bohrweg 2, 2333 CA Leiden, The Netherlands
}

Accepted XXX. Received YYY; in original form ZZZ

\begin{abstract}
We report the discovery of a nearby dwarf galaxy in the constellation of Hydrus, between the Large and the Small Magellanic Clouds. Hydrus 1 is a mildy elliptical ultra-faint system with luminosity $M_{V} \sim-4.7$ and size $\sim 50 \mathrm{pc}$, located $28 \mathrm{kpc}$ from the Sun and $24 \mathrm{kpc}$ from the LMC. From spectroscopy of $\sim 30$ member stars, we measure a velocity dispersion of $2.7 \mathrm{~km} \mathrm{~s}^{-1}$ and find tentative evidence for a radial velocity gradient consistent with $3 \mathrm{~km} \mathrm{~s}^{-1}$ rotation. Hydrus 1's velocity dispersion indicates that the system is dark matter dominated, but its dynamical mass-to-light ratio $\mathrm{M} / \mathrm{L} \sim 66$ is significantly smaller than typical for ultra-faint dwarfs at similar luminosity. The kinematics and spatial position of Hydrus 1 make it a very plausible member of the family of satellites brought into the Milky Way by the Magellanic Clouds. While Hydrus 1's proximity and well-measured kinematics make it a promising target for dark matter annihilation searches, we find no evidence for significant gamma-ray emission from Hydrus 1. The new dwarf is a metal-poor galaxy with a mean metallicity $[\mathrm{Fe} / \mathrm{H}]=-2.5$ and $[\mathrm{Fe} / \mathrm{H}]$ spread of 0.4 dex, similar to other systems of similar luminosity. Alpha-abundances of Hyi 1 members indicate that star-formation was extended, lasting between 0.1 and $1 \mathrm{Gyr}$, with self-enrichment dominated by SN Ia. The dwarf also hosts a highly carbon-enhanced extremely metal-poor star with $[\mathrm{Fe} / \mathrm{H}] \sim-3.2$ and $[\mathrm{C} / \mathrm{Fe}] \sim+3.0$.
\end{abstract}

Key words: Magellanic Clouds - galaxies: dwarf - Galaxy: halo - Galaxy: globular clusters: general - Galaxies: Local Group - stars: general

\section{INTRODUCTION}

There is no solution to the "missing satellites" problem if it is defined simply as the mismatch between the total count

*This paper includes data gathered with the 6.5 meter Magellan Telescopes located at Las Campanas Observatory, Chile.

†E-mail: skoposov@cmu.edu of the Milky Way dwarf galaxies and the predicted number of dark matter halos capable of hosting a galaxy, since there is no problem. Once the observed number of the Galactic satellites is scaled up to correct for the effects of the detection efficiency and the number of DM sub-halos is scaled down in accordance with our understanding of the physics of star-formation, little inconsistency remains (Benson et al. 2002; Koposov et al. 2008; Tollerud et al. 2008; Koposov et 
al. 2009; Jethwa et al. 2018). However, to make the theory and the observations match requires that the vast majority of the Galactic satellites have extremely low luminosities but occupy sub-halos with significant virial masses. Only in the past 15 years, thanks to the plethora of imaging surveys such as Sloan Digital Sky Survey (York et al. 2000), PanSTARRS (Kaiser et al. 2002), VST ATLAS (Shanks et al. 2015) and the development of automated search techniques (e.g. Willman et al. 2005a; Belokurov et al. 2007; Koposov et al. 2008; Walsh et al. 2009), the satellite census has been expanded to include systems with luminosities of $L \sim 100 L \odot$ estimated to inhabit DM halos of at least $\sim 10^{7}-10^{9} M_{\odot}$ (e.g. Simon \& Geha 2007; Koposov et al. 2009; Jethwa et al. 2018). Thus, the "missing satellites" problem is perhaps better viewed as a dramatic divergence between the sub-halo mass function and the dwarf luminosity function (Klypin et al. 1999), with a pile-up of literally invisible objects with mass-to-light ratios of order $10^{5}-10^{6} M_{\odot} L_{\odot}^{-1}$.

The faintest of these ultra-faint dwarfs are only observable in and around the Milky Way, thus making the Galaxy a unique place to provide crucial constraints on theories of Universal structure formation. However, as a result of the expansion of the discoveries into the ultra-faint regime, one problem appears to have emerged. Since most searches have a limiting surface brightness of $30-32$ mag $\operatorname{arcsec}^{-2}$, many of the recently detected systems tend to have small physical sizes, often only a few tens of parsecs. This makes the classification into dwarf galaxies and globular clusters ambiguous based on the object's size alone (Gilmore et al. 2007; Martin et al. 2008). Furthermore, the dynamical tests for the presence of dark matter in the faint systems are often fraught with difficulties caused by low numbers of member stars accessible for spectroscopy (Walker et al. 2009c), stellar binaries (Koposov et al. 2011) and instrument systematics (Simon \& Geha 2007). As a result, some low luminosity satellites have oscillated between the two classes (e.g. Belokurov et al. 2014; Laevens et al. 2014; Bonifacio et al. 2015; Weisz et al. 2016; Voggel et al. 2016) while the nature of some remains uncertain today (Willman et al. 2005b, 2011). To circumnavigate these displeasing ambiguities, additional diagnostics of "galaxyness" have been offered, including, in particular, one relying on the presence of a spread of heavy element abundances (see Willman \& Strader 2012) indicative of a prolonged star formation activity.

Most recently, our view of the Milky Way satellite population has become even more curious. In the past 3 years, data from the various observing campaigns utilizing the Dark Energy Camera - such as DES, MagLiteS (DrlicaWagner et al. 2016), and SLAMS (Jethwa et al. 2017) - have yielded a stream of new satellite discoveries (Koposov et al. 2015a; Bechtol et al. 2015; Kim et al. 2015; Kim \& Jerjen 2015; Martin et al. 2015; Drlica-Wagner et al. 2015b). Many of these have been uncovered thanks to the superb quality of deep DECam data that has facilitated detection of fainter and more distant systems. However, many were brought to light simply due to the expansion of the imaging surveys into the previously barely explored Southern celestial hemisphere. Remarkably, the bulk of the Southern discoveries are situated near the Magellanic Clouds (Koposov et al. 2015a), which in hindsight seems obvious. Initially, as the data started to come in, the satellite over-density signal was barely significant. However, at present, the satellite positions (Drlica-Wagner et al. 2016; Torrealba et al. 2018) and their kinematics (Koposov et al. 2015b; Walker et al. 2016) appear to favor the hypothesis in which the Magellanic Clouds (MCs) have brought a large group of their smaller companions into the Milky Way (Deason et al. 2015; Jethwa et al. 2016). At the same time, thanks to a deeper and more complete view of the Magellanic area, supplied by the DECam and Gaia data, we have learned a great deal about the Clouds themselves and their interaction with each other (see Mackey et al. 2017; Belokurov et al. 2017).

Around the Clouds, the relatively-unexplored portion of the sky that has the greatest potential to inform our understanding of both the satellites of the Magellanic satellites and the Clouds' life as a pair is the area directly between the LMC and the SMC. This paper presents results from a continuation of the survey of the Magellanic bridge region, first presented by Mackey et al. (2017). While this survey was motivated primarily by the study of the stellar tidal debris field around the Magellanic Clouds (see e.g. Mackey et al. 2016; Belokurov \& Koposov 2016), it also provides a great resource to trawl for new MC satellites. Here we report the discovery and a detailed photometric, spectroscopic and chemical study of exactly that: a new dwarf galaxy in the bridge region of the Magellanic Clouds. The paper is structured as follows. In Section 2 we describe the photometric data from Dark Energy Camera. In Section 3 we discuss the satellite properties that we infer from the photometric data. In Section 4 we discuss the spectroscopic data and modeling of a new satellite. Section 5 provides a discussion of all the chemical, dynamical and morphological properties of the new system, possible connections to the Magellanic Clouds and prospects for dark matter annihilation searches. The Section 6 concludes the paper. The properties of the stellar debris field around the Magellanic Clouds are discussed in a companion paper by Mackey et al (2018).

\section{PHOTOMETRIC DATA}

This work utilizes the photometric data from two DECam programs, 2016A-0618 and 2017B-0906, focusing on the region between the Magellanic Clouds. The first results based on the 2016A-0618 imaging have been presented in Mackey et al. (2017). The DECam is a large (570 mega-pixel) mosaic camera boasting 62 science CCDs, giving a combined field-of-view of 3 square degrees (Flaugher et al. 2015). The camera is installed on the 4-m Blanco Telescope located at Cerro-Tololo Inter-American Observatory (CTIO) in Chile. The camera is equipped with ugrizY filters and has been used for the last 6 years to conduct the Dark Energy Survey (The Dark Energy Survey Collaboration 2005; Abbott et al. 2018) as well as a number of individual programs and minisurveys (Drlica-Wagner et al. 2016; Schlafly et al. 2018).

The data for the program 2017B-0906 - the primary data source for this paper - were acquired on 8 and 9 October 2017. We observed the area covering the side of the Magellanic bridge region closest to the Small Magellanic Cloud. Images were taken in $g$ and $r$ filters with exposure times ranging from 70 to $225 \mathrm{~s}$ in the $\mathrm{g}$ band and from 60 to 110 $\mathrm{s}$ in the $\mathrm{r}$ band, where the variation was due to changing lunar illumination during the night. On average, in the survey, 
each sky location was covered by 3 individual exposures and the total observed area is approximately 140 square degrees.

The initial data processing was carried out using the community pipeline (Valdes et al. 2014) which provided us with calibrated single exposure images that were corrected for bias, flat-field, cross-talk, nonlinearity as well as being WCS-aligned and remapped. The subsequent image processing was very similar to that described by Mackey et al. (2017). We first created image stacks in $g$ and $r$ bands from all of the available data using the SWARP software (Bertin 2010). The sources extracted from each CCD frame were used to construct the Point Spread Function (PSF) by running PSFex (Bertin 2011). Then we used a custom version of the SExtractor code (Bertin \& Arnouts 1996) in double image mode on stacked and individual images to obtain uncalibrated forced photometry PSF fluxes from individual images. The fluxes were then photometrically calibrated and averaged for sources with more than one observation. The lists of detected $g$ and $r$-band sources were cross-matched to produce the final catalogue with a magnitude limit of $\sim 23$ in both $g$ and $r$.

The photometric calibration was done using the APASS DR9 catalogue (Henden et al. 2012). Because APASS has significant spatially dependent systematic errors reaching up to $0.05-0.1 \mathrm{mag}$, we first rectified the APASS magnitudes using Gaia (Gaia Collaboration et al. 2016a,b) and 2MASS (Skrutskie et al. 2006) data. To perform the APASS correction, we assumed that the APASS $g$ and $r$ magnitude for stars with $0<J-K_{s}<0.75$ could be estimated from the Gaia $G$ band measurement and the polynomial function of the 2MASS $J-K_{s}$ colour. This allowed us to compute the $g$ and $r$ magnitude HEALPIX (Górski et al. 2005) correction map for APASS with resolution of $N_{\text {side }}=128$ (corresponding to $\sim 0.5^{\circ} \times 0.5^{\circ}$ degrees). Finally we brought the magnitudes from the APASS photometric system into the DES DR1 system (Abbott et al. 2018) using simple color-terms. The recalibrated APASS magnitudes together with the information from the overlapping DECam exposures were then used to establish zero-points for each DECAM exposure as well as the CCD-specific zero-point offsets (similarly to Koposov et al. (2015a)). A comparison of the final calibrated catalogue with the small patches of the DES DR1 that overlap with our footprint reveals that the remaining systematic errors in the catalogue are at a level $\lesssim 0.03 \mathrm{mag}$.

Throughout the paper we use extinction corrected magnitudes based on the Schlegel et al. (1998) extinction model with the coefficients from Schlafly \& Finkbeiner (2011). To separate stars from galaxies in our catalogue we use the criterion based on the SPREAD_MODEL parameter measured by Sextractor in the $r$ band: $\left|S P R E A D \_M O D E L\right|<0.003$ (Desai et al. 2012).

In order to ensure a uniform calibration for both of our datasets, 2016A-0618 and 2017B-0906, we reprocessed the data from 2016 together with data from 2017 to obtain a continuous coverage of $\sim 380$ square degrees across the inter-Cloud region. Figure 1 shows the density distribution of distant $20.5<r<220<g-r<0.3$ main sequence turnoff (MSTO) stars selected from our two Magellanic Cloud programs as well as the data from the Dark Energy Survey Data Release 1. The combined footprint of our programs is traced by a blue contour showing the targeted region of sky. We remark that the data do not show any discontinu-

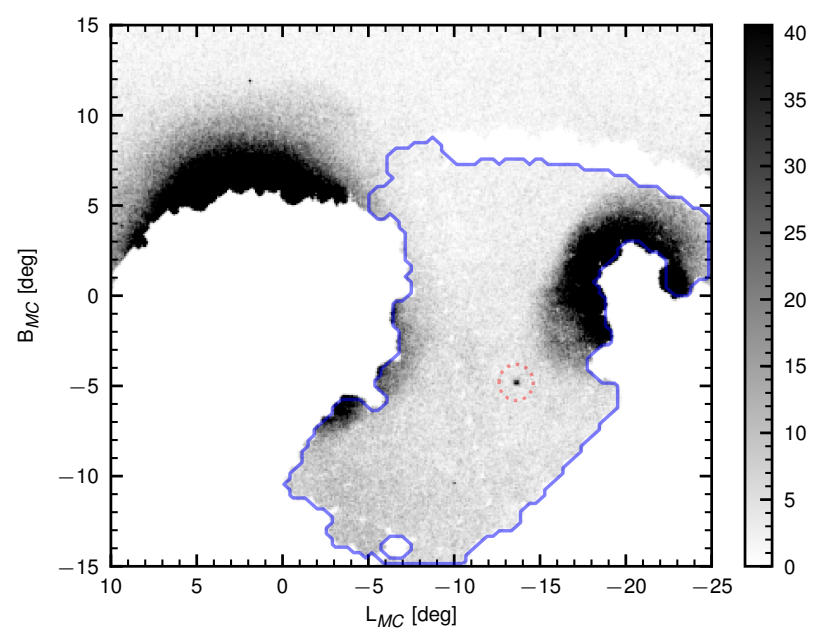

Figure 1. The density of MSTO stars $(20.5<r<22$ and $0<g-$ $r<0.3$ in the Magellanic Clouds region. We include data from our new DECam observing program, data from Mackey et al. (2017) and data from the Dark Energy Survey DR1. The data from our Magellanic bridge observations is surrounded by blue contour. The location of newly discovered dwarf galaxy in between Large Magellanic Cloud (on the left) and Small Magellanic Cloud (on the right) is marked by a red dashed circle. The coordinate system used for the plot is a rotated spherical coordinate system such that the equator is going through both LMC and SMC centers. The pole of this coordinate system is $\alpha_{\text {pole }}, \delta_{\text {pole }}=39.61^{\circ}, 15.49^{\circ}$ and the longitude zeropoint is at the location of LMC.

ity either between our 2016 and 2017 data or between our program and the DES survey, thus confirming the absence of significant systematic offsets in our photometry.

A stand-alone feature immediately apparent in the stellar density map in Figure 1 is an overdensity at $L, B=$ $(-13,-5)$. This overdensity in the Hydrus ${ }^{1}$ constellation is the subject of this paper. In what follows we classify the satellite as a dwarf galaxy (see Section 4) and therefore it is referred to as Hydrus 1 or Hyi 1 in the rest of the text.

\section{SATELLITE PROPERTIES}

\subsection{Stellar population}

We start by analyzing the color-magnitude diagram (CMD) of the overdensity circled in red in Figure 1. The left panel of Figure 2 shows the background-subtracted Hess diagram of the central $10^{\prime}$ of the object. A clear main sequence (MS) together with a part of the red giant branch are both readily visible, confirming that the object is a bona-fide satellite. The Hess diagram of the overdensity is also clearly different from the color-magnitude distribution of foreground stars around it shown in the right panel of the Figure. To measure the distance to the satellite and constrain its stellar population properties, we perform a fit to the Hess diagram of the system within the inner $10^{\prime}$ by using two different sets

1 The Hydrus constellation is different from Hydra. Hydrus is a mythical creature usually represented by a male water snake. 


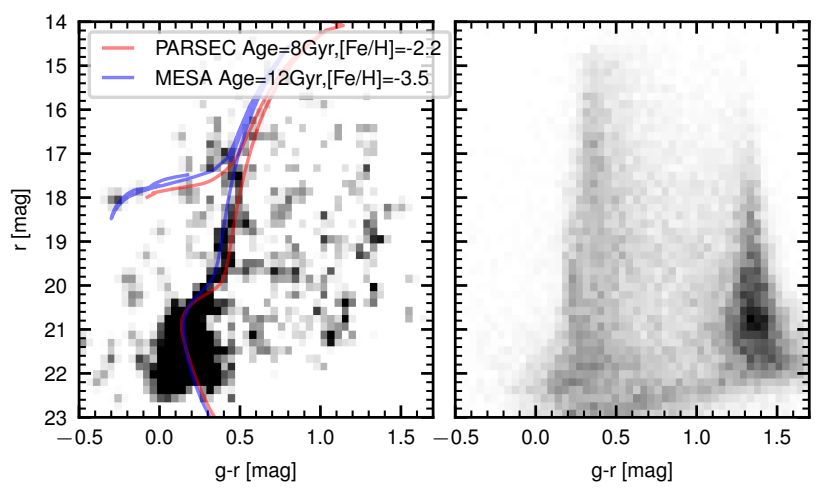

Figure 2. Left panel: Background subtracted $g-r, r$ Hess diagram within $10^{\prime}$ of Hydrus 1. Stars within an annulus with radii of $20^{\prime}$ and $60^{\prime}$ were used for the background. Two theoretical isochrones shifted to a distance of $31 \mathrm{kpc}$ from MESA and PARSEC libraries are overplotted in blue and red respectively. Right panel: Hess diagram of background stars around Hydrus 1 (in 20',60' annulus).

of theoretical isochrones. More precisely, we use both PARSEC isochrones (Bressan et al. 2012; Marigo et al. 2017) ${ }^{2}$ and MESA isochrones Dotter (2016); Choi et al. (2016); Paxton et al. (2011). The isochrones are populated according to the Chabrier Initial Mass Function (IMF) (Chabrier 2003) with an additional magnitude-dependent photometric scatter based on the uncertainties in the data. We then evaluate the likelihood of the observed CMD over a grid of ages, metallicities and heliocentric distances. The resulting best-fit MESA and PARSEC isochrones are overplotted in the left panel of the Figure 2.

The best parameters for the MESA isochrone are: age of $12 \mathrm{Gyr}$, iron abundance of $[\mathrm{Fe} / \mathrm{H}]=-3.5$ and distance of $31 \mathrm{kpc}$. On the other hand, for the PARSEC isochrone we obtain $8 \mathrm{Gyr},[\mathrm{Fe} / \mathrm{H}]=-2.2$ and distance of $\sim 31 \mathrm{kpc}$. We find that MESA isochrones fit the observed Hess diagram somewhat better, possibly because the data are pushing the PARSEC library to its lower limit of $[\mathrm{Fe} / \mathrm{H}]=-2.2$. Due to large systematic differences in isochrone solutions we do not try to assess the uncertainties in the age and metallicites inferred, however the formal uncertainty on the heliocentric distance for each isochrone set is around $2 \mathrm{kpc}$. While CMD fitting is an acceptable method of deriving distances it is also known to suffer from inaccuracies associated with the age-metallicity-distance degeneracies as well as biases associated with the isochrone libraries used. These issues can be mitigated by using horizontal branch stars (both pulsating and not) which can offer better accuracy in distance in most cases. In fact in Figure 2 we can see $\sim 4$ stars at $\mathrm{r} \sim 18$ and $\mathrm{g}-\mathrm{r} \lesssim 0$ that are likely Hydrus's Blue Horizontal Branch (BHB) stars (some of these were later spectroscopically confirmed; see Section 4). This group of four likely BHB stars can be used to measure the distance by applying the fiducial relation for the horizontal branch from Deason et al. (2011). As that relation was defined for the SDSS photometric sys-

2 We use the PARSEC v1.2S,COLIBRI PR16 isochrones downloaded from http://stev.oapd.inaf.it/cgi-bin/cmd in February 2018 tem, we recalibrate it into the DES system. The resulting equation that we adopt is:

$$
M_{g}=0.444+0.106 c+0.704 c^{2}+1.126 c^{3}+0.794 c^{4}
$$

where $c=(g-r) / 0.3$. With four BHB stars we derive the distance modulus $m-M=17.20 \pm 0.04$ or distance of $D=$ $27.6 \pm 0.5 \mathrm{kpc}$, which is roughly consistent with the isochrone fit result. The expected systematic uncertainty on the BHB measurement should be of the order of $10 \%$ (Fermani \& Schönrich 2013).

To validate the distance measurement we have employed the Magellanic RR Lyrae catalog from the OGLE-IV project (Udalski et al. 2015) which covers the location of Hydrus 1. Somewhat suprisingly, within twice the half-light radius of Hyi 1 we identified two RRab Lyrae stars, OGLE-SMCRRLYR-6316 and OGLE-SMC-RRLYR-6325, with distance moduli of 17.22 and 17.19 respectively $^{3}$. With an average

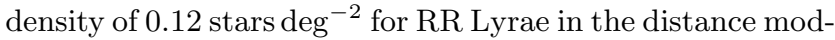
ulus range $17<m-M<17.4$, the probability of having two Galactic RR Lyrae within twice the half light radius of Hyi 1 is $P \approx 2 \times 10^{-4}$. This suggests that both RR lyrae are most likely genuine members of Hyi 1 . The RR Lyrae fully corroborate the distance estimate of $m-M=17.2$ that we obtained based on BHB magnitudes.

With period and V-band amplitude combination of $(0.67 \mathrm{~d}, 0.8)$ and $(0.73 \mathrm{~d}, 0.56)$, the two RR Lyrae in Hyi 1 are of Oosterhoff type II. This is in perfect agreement with the recent discovery that the fraction of Oo I/Oo II types depends strongly on the stellar mass of the host (see e.g. Fiorentino et al. 2015; Belokurov et al. 2018), with higher luminosity objects (e.g. massive dwarf galaxies) containing a larger fraction of Oo I RRab stars. Extended down to the typical luminosities of UFDs, this trend predicts that the RR Lyrae contents of objects like Hyi 1 should be almost entirely composed of Oo II type pulsators.

\subsection{Spatial distribution}

This section looks in detail at the spatial distribution of stars inside the Hydrus 1 satellite. Figure 3 gives the positions of the MSTO stars with $0<g-r<0.3,20.5<r<22$ in 1 square degree region around the center of the satellite. We clearly see a high-contrast over-density with some hint of elongation approximately along the right ascension direction. In the Figure we also overplot the locations of the BHB stars and the RR-lyrae discussed in the previous section, thus confirming that they are clustered near the center of Hydrus 1. To describe the morphological properties of Hyi 1, we model the stellar distribution with a combination of uniform background and flattened exponential profile (similar to Koposov et al. 2015a). We adopt standard priors on the model parameters: a Jeffrey's prior on the exponential scale length, uniform $U(0,1)$ priors on the object ellipticity and the fraction of stars belonging to Hydrus 1 , a $U(0,180)$ prior on the position angle and an improper uniform prior on the position of the center of the object. We then sample

3 To compute distances to the RR Lyrae we use the periodluminosity relations from Catelan et al. (2004), metallicity phase relation from Smolec (2005) as prescribed by Pietrukowicz et al. (2015) 


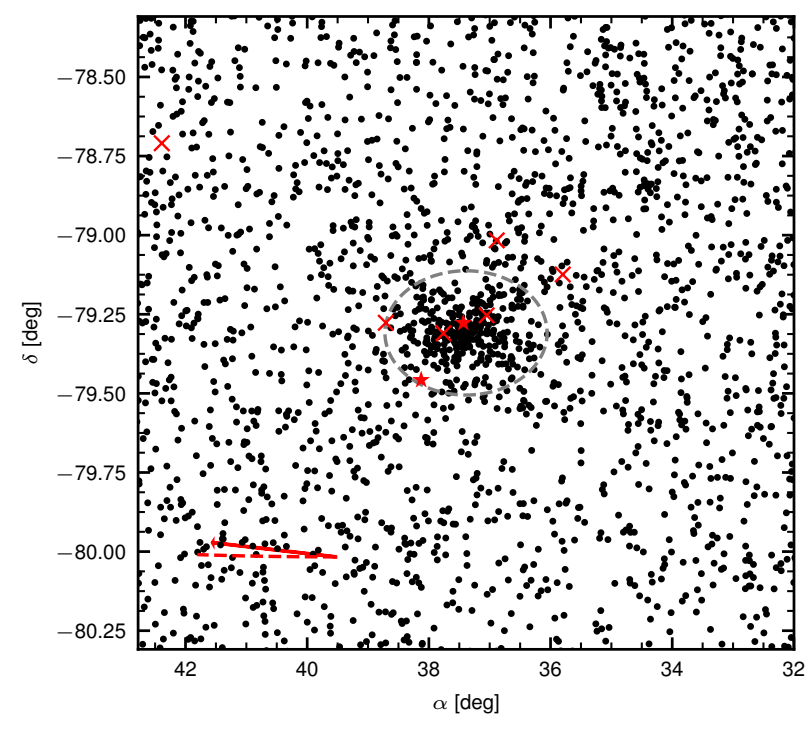

Figure 3. The distribution of MSTO $(0<g-r<0.3,20.5<$ $r<22)$ stars in the vicinity of the Hydrus 1 satellite. The dashed grey ellipse shows the best-fit shape of the satellite where the major axis of the ellipse is twice the half-light radius. The arrow in the left corner shows the direction of the LMC's proper motion and the dashed line is parallel to the line connecting LMC and SMC. Red crosses show the locations of BHB candidate stars with distance modulus within 0.1 magnitude of Hydrus 1, while red star symbols identify the locations of RR Lyrae stars with distance modulus within 0.1 magnitudes of Hydrus 1. We note that both the RR Lyrae and BHB stars show clear concentrations near the center of the object.

the posterior using a Markov Chain Monte-Carlo (MCMC) technique, specifically with the ensemble sampler (Goodman \& Weare 2010) as implemented by Foreman-Mackey et al. (2013). The summary of our measurements is provided in Table 1. Here and throughout the rest of the paper, when we report the measurements and their associated uncertainties we use the mean and standard deviation in the case of symmetric Gaussian-like posteriors and the median and 15.8 and 84.2 percentiles for asymmetric posteriors. In the Table we report both the major-axis half-light radius (i.e $1.67 \mathrm{~h}$ where $h$ is the exponential scale-length along the major axis) as well as the circularized half-light radius (i.e. multiplied by $\sqrt{1-e})$.

The angular half-light radius of the satellite is $\sim 6.6^{\prime}$ while its physical size, assuming a distance of $28 \mathrm{kpc}$, is $\sim$ 50 pc. These values suggest that Hyi 1 is a dwarf galaxy rather than a globular cluster (see e.g. Figure 6 of Torrealba et al. 2016). We observe that the ellipticity of the system is significantly different from zero: $e=1-\frac{b}{a} \sim 0.2$, confirming its slightly elongated visual appearance in Figure 3 . It is worth noting that the elongation of the system is approximately aligned with the Magellanic bridge and the proper motion of the Magellanic Clouds (shown as a red dashed line segment and a red arrow, respectively, in Fig. 3). For now, we refrain from speculation about whether this is a mere coincidence or a tell-tale sign of a connection between Hydrus 1 and the Magellanic Clouds. To verify whether the exponential model adopted for Hyi 1's density profile pro-

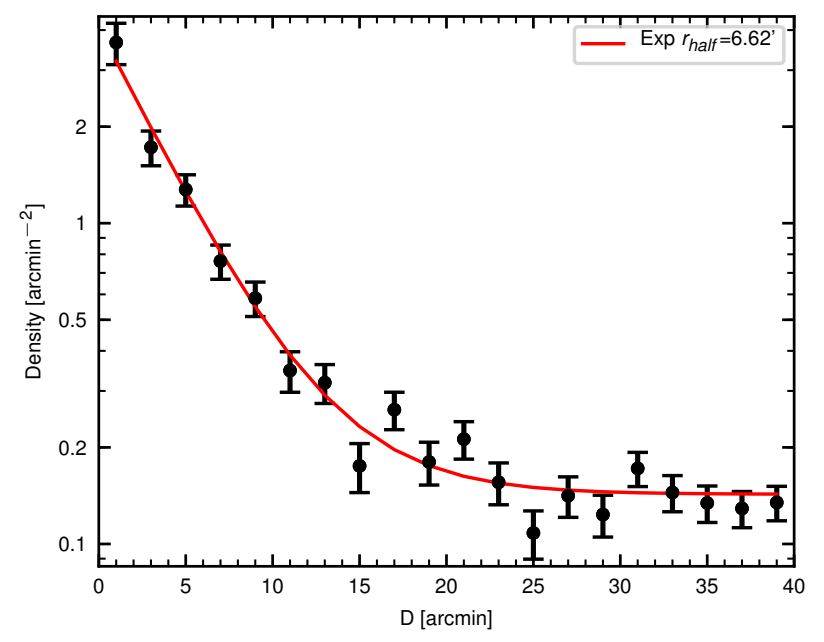

Figure 4. Azimuthally averaged density profile of Hydrus 1 turnoff stars with $0<g-r<0.3,20.5<r<22.25$. The red curve shows our best fit exponential model.

vides an accurate description of the data, Figure 4 displays the azimuthally averaged 1-D density profile of the satellite together with the best-fit exponential model. Reassuringly, the model reproduces the observed profile well at all radii.

Equipped with a color-magnitude model and a spatial model of the satellite we can proceed to measure the absolute luminosity of the system. We first compute the number of stars in Hydrus 1 that are near our best-fit isochrone and brighter than $r=21.75$. This is approximately 1 magnitude above the limit of our photometric data and therefore should give us an almost complete sample. Above this limit Hyi 1 has an estimated $387 \pm 28$ stars, which, assuming the bestfit MESA isochrone at the distance from Section 3.1 and a Chabrier IMF, gives a total stellar mass of $\sim 6000 \mathrm{M}_{\odot}$ and an expected V-band absolute luminosity of $-4.74 \pm 0.08^{4}$. We also checked that using the PARSEC isochrone yields the same absolute magnitude within the uncertainty.

\section{SPECTROSCOPY}

In order better understand the nature of Hydrus 1, we acquired spectra of its brightest member stars.

\subsection{Target selection}

We selected potential members of the Hyi 1 system as those that lie spatially near the center of Hyi 1 and photometrically near the best-fitting isochrone measured in Section 3.1. The leftmost panel of Figure 5 shows the spatial distribution of all 235 spectroscopic targets (grey and black points), and

\footnotetext{
4 We remark that the total luminosity that we compute is the expected luminosity given the total stellar mass and mass function, rather than the estimate obtained by summing luminosities of individual stars, which tends to suffer from large fluctuations due to poorly populated red giant branch.
} 


\begin{tabular}{|c|c|c|c|}
\hline Parameter & Description & Value & Unit \\
\hline$\alpha_{c e n}$ & Right ascension of the center & $37.389 \pm 0.031$ & degree \\
\hline$\delta_{c e n}$ & Declination the center & $-79.3089 \pm 0.0045$ & degree \\
\hline$r_{\text {half }, m a j}$ & Major axis half light radius & $7.42_{-0.54}^{+0.62}$ & $\operatorname{arcmin}$ \\
\hline$r_{h a l f, m a j} \sqrt{1-e}$ & Circularised half-light radius & $6.64_{-0.43}^{+0.46}$ & $\operatorname{arcmin}$ \\
\hline$r_{h a l f, \operatorname{maj}} \sqrt{1-e}$ & Circularised half-light radius & $53.3 \pm 3.6$ & $\mathrm{pc}$ \\
\hline $1-b / a$ & Ellipticity & $0.21_{-0.07}^{+0.15}$ & \\
\hline PA & Positional angle of the major axis of stellar distribution & $97 \pm 14$ & degree \\
\hline $\mathrm{m}-\mathrm{M}$ & Distance modulus & $17.20 \pm 0.04$ & \\
\hline Distance & Heliocentric distance & $27.6 \pm 0.5$ & $\mathrm{kpc}$ \\
\hline$M_{V}$ & Absolute Magnitude & $-4.71 \pm 0.08$ & mag \\
\hline$\sigma_{\text {los }}$ & Line of sight velocity dispersion & $2.69_{-0.43}^{+0.51}$ & $\mathrm{~km} \mathrm{~s}^{-1}$ \\
\hline$V_{h e l}$ & Mean Heliocentric radial velocity & $80.4 \pm 0.6$ & $\mathrm{~km} \mathrm{~s}^{-1}$ \\
\hline$V_{G S R}$ & Mean radial velocity in the Galactic rest frame ${ }^{5}$ & $-94.1 \pm 0.6$ & $\mathrm{~km} \mathrm{~s}^{-1}$ \\
\hline $\mathrm{M}\left(\mathrm{r}<\mathrm{r}_{1 / 2}\right)$ & Total dynamical mass inside half-light radius ${ }^{6}$ & $2.23_{-0.66}^{+0.95} 10^{5}$ & $M_{\odot}$ \\
\hline $\mathrm{M} / \mathrm{L}\left(\mathrm{r}<\mathrm{r}_{1 / 2}\right)$ & Mass-to-light ratio inside the half-light radius & $66_{-20}^{29}$ & $\mathrm{M}_{\odot} / \mathrm{L}_{\odot}$ \\
\hline $\log _{10} \mathrm{M} / \mathrm{L}\left(\mathrm{r}<\mathrm{r}_{1 / 2} /\left(M_{\odot} / L_{\odot}\right)\right)$ & Logarithm of mass-to-light ratio inside the half-light radius & $1.82 \pm 0.16$ & dex \\
\hline $\log _{10}\left[J\left(0.5^{\circ}\right) /\left(\mathrm{GeV}^{2} \mathrm{~cm}^{-5}\right)\right]$ & Logarithm of the $J$ factor & $18.33_{-0.34}^{+0.38}$ & dex \\
\hline$\frac{d V_{h e l}}{d \alpha \cos \delta_{c e n}}$ & Radial velocity gradient along right ascension & $24.4 \pm 9.6$ & $\mathrm{~km} \mathrm{~s}^{-1} \mathrm{deg}^{-1}$ \\
\hline$\frac{d V_{h e l}}{d \delta}$ & Radial velocity gradient along declination & $4.1 \pm 9.2$ & $\mathrm{~km} \mathrm{~s}^{-1} \mathrm{deg}^{-1}$ \\
\hline$<[\mathrm{Fe} / \mathrm{H}]>$ & Mean spectroscopic metallicity & $-2.52 \pm 0.09$ & dex \\
\hline$\sigma_{[\mathrm{Fe} / \mathrm{H}]}$ & Spectroscopic metallicity scatter & $0.41 \pm 0.08$ & $\operatorname{dex}$ \\
\hline
\end{tabular}

Table 1. Key photometric and kinematic properties of the Hydrus 1 dwarf galaxy. Reported measurements and uncertainties are either means and standard deviations for symmetric posteriors, or medians and 15.8 and 84.2 percentiles in the case of asymmetric posteriors.

the second panel from the left shows the extinction corrected color-magnitude distribution of those same stars.

\subsection{Spectroscopic data}

On 12-13 Feb 2018, we used M2FS, a multi-object fiber spectrograph at the Magellan Clay $6.5 \mathrm{~m}$ telescope, to acquire spectra for the selected targets. We used the same instrument configuration as in our previous M2FS observations of ultra-faint dwarf galaxy candidates (Walker et al. 2015, 2016) - specifically, we observed the spectral region 5130 $5190 \AA$ at resolution $\mathcal{R} \sim 18,000$. We obtained $3 \times 2700$ second exposures (the middle exposure lost $\sim 300$ seconds due to technical problems) during conditions that were clear, with good seeing but high airmass $(\sim 1.7-2.0)$. For calibration purposes, we also obtained high-S/N exposures of the solar spectrum during evening twilight, as well as Th-Ar arc-lamp images immediately before and after the science exposures.

We processed and fit stellar-atmospheric models to all spectra following procedures identical to those described by Walker et al. (2015, 2016). For each star, we obtain simultaneous estimates of line-of-sight velocity, effective temperature, surface gravity and metallicity. For all the parameters we adopt uninformative flat priors with the exception of the effective temperature, for which we use a log-normal prior based on the $g-r$ color and spectroscopic metallicity. Our prior takes the form of $\log T_{\text {eff }} \sim$ $\mathcal{N}\left(S_{1}(g-r)+A \exp (B[F e / H]), S_{2}(g-r)\right)$ where A, B are fitted constants and $S_{1}(g-r)$ and $S 2(g-r)$ are cubic splines

${ }^{5}$ Computed assuming the $V_{L S R}=232.8 \mathrm{~km} \mathrm{~s}^{-1}$ from McMillan (2017) and peculiar solar velocity from Schönrich et al. (2010)

6 Obtained using the formula of Walker et al. (2009b) with 20 equidistant knots in the $-0.3<g-r<1.2$ range. We calibrate this prior using the cross-match of the Dark Energy Survey DR1 photometric catalogue with the SDSS DR9 spectroscopic catalogue, where the $g-r$ colors were taken from DES and metallicities and effective temperatures from measurements by SDSS Spectroscopic Parameter Pipeline (Lee et al. 2008a,b; Allende Prieto et al. 2008). The median parameter uncertainties for the spectra with signal to noise larger than three are $\sigma_{v}=0.82 \mathrm{~km} \mathrm{~s}^{-1}, \sigma_{\text {Teff }}=80 \mathrm{~K}$, $\sigma_{\operatorname{logg}}=0.19 \mathrm{dex}$ and $\sigma_{\mathrm{Fe} / \mathrm{H}}=0.13 \mathrm{dex}$. The velocity uncertainties include a systematic floor, added in quadrature, of $0.2 \mathrm{~km} \mathrm{~s}^{-1}$, as measured from repeated observations of twilight spectra.

Of the 235 spectroscopically observed stars, 117 have signal-to-noise larger than 3 and 136 stars have radial velocity (RV) uncertainty less than $10 \mathrm{~km} \mathrm{~s}^{-1}$. As large RV uncertainties of $10 \mathrm{~km} \mathrm{~s}^{-1}$ and more occur with our highresolution spectra only when the spectrum lacks discernable spectral features and/or has too low signal to noise, we exclude all the stars with $\sigma_{v}>10 \mathrm{~km} \mathrm{~s}^{-1}$ from further analysis. We show these stars in the second panel of Figure 5 by empty circles. As expected, most of these stars are faint and have magnitudes $r \gtrsim 19.5$, with the exception of two blue/hot stars that are likely BHBs that lack strong features within the observed wavelength range.

Table 2 presents spectroscopic parameter measurements for all the stars that either have signal-to-noise larger than 3 or have radial velocity uncertainty less than $10 \mathrm{~km} \mathrm{~s}^{-1}$. We list measurements of the effective temperature, surface gravity, metallicity and heliocentric line-of-sight velocitiy. We also provide in the table the $g, r$ magnitudes and the log-odds of being Hydrus 1 member star, based on the model described in the next Section.

The right three panels of Figure 5 summarize our spec- 


\begin{tabular}{|c|c|c|c|c|c|c|c|c|c|c|c|c|c|c|}
\hline ID & $\begin{array}{c}\alpha \\
\text { degree }\end{array}$ & $\begin{array}{c}\delta \\
\text { degree }\end{array}$ & $S / N$ & $\begin{array}{c}V_{h e l} \\
\mathrm{~km} \mathrm{~s}^{-1}\end{array}$ & $\begin{array}{c}\sigma_{V} \\
\mathrm{~km} \mathrm{~s}\end{array}$ & $\begin{array}{c}\mathrm{T}_{\text {eff }} \\
\mathrm{K}\end{array}$ & $\begin{array}{c}\sigma_{T, \text { eff } f} \\
\mathrm{~K}\end{array}$ & $\log g$ & $\sigma_{\log g}$ & $\begin{array}{c}{[\mathrm{Fe} / \mathrm{H}]} \\
\operatorname{dex}\end{array}$ & $\begin{array}{c}\sigma_{[F e / H]} \\
\operatorname{dex}\end{array}$ & $\ln \frac{P_{m}}{P_{n m}}$ & $\begin{array}{c}\mathrm{g} \\
\mathrm{mag}\end{array}$ & $\begin{array}{c}\mathrm{r} \\
\mathrm{mag}\end{array}$ \\
\hline 2 & 37.69 & -79.36 & 15.4 & 53.5 & 0.5 & 5723 & 64 & 4.53 & 0.12 & -0.74 & 0.06 & $-64.1_{-24.7}^{+17.3}$ & 18.45 & 17.92 \\
\hline 3 & 37.70658 & -79.35572 & 21.7 & 5.0 & 0.5 & 5353 & 35 & 4.49 & 0.08 & -0.7 & 0.04 & $-415.8_{-169.4}^{+119.8}$ & 17.86 & 17.25 \\
\hline 4 & 37.74029 & -79.35434 & 9.7 & 83.1 & 1.7 & 5521 & 82 & 1.4 & 0.46 & -2.34 & 0.15 & $7.0_{-0.9}^{+1.0}$ & 19.03 & 18.44 \\
\hline 5 & 37.694 & -79.34884 & 6.0 & 211.9 & 1.1 & 5904 & 114 & 1.16 & 0.84 & -1.75 & 0.2 & $-1168.4_{-488.1}^{+343.8}$ & 19.74 & 19.24 \\
\hline 6 & 37.72267 & -79.33451 & 18.1 & -6.1 & 0.5 & 5437 & 33 & 4.81 & 0.1 & -0.54 & 0.04 & $-545.6_{-224.0}^{+157.1}$ & 18.21 & 17.63 \\
\hline 7 & 37.69496 & -79.32996 & 2.1 & 243.7 & 2.9 & 6269 & 240 & 3.35 & 0.86 & -0.9 & 0.45 & $-1812.6_{-755.8}^{+533.2}$ & 20.89 & 20.45 \\
\hline 10 & 37.79812 & -79.40667 & 4.7 & 83.9 & 1.5 & 5876 & 111 & 4.52 & 0.29 & -1.58 & 0.19 & $1.3_{-3.0}^{+1.6}$ & 19.81 & 19.29 \\
\hline 11 & 37.83383 & -79.38515 & 10.0 & 6.3 & 0.6 & 5792 & 91 & 3.18 & 0.19 & -1.27 & 0.11 & $-396.6_{-164.5}^{+116.7}$ & 18.84 & 18.33 \\
\hline 12 & 37.75471 & -79.349 & 3.7 & 187.4 & 1.5 & 5502 & 111 & 2.84 & 0.97 & -1.88 & 0.27 & $-765.2_{-321.1}^{+227.0}$ & 20.27 & 19.66 \\
\hline 14 & 37.81337 & -79.32914 & 2.2 & 85.7 & 6.5 & 6387 & 230 & 2.86 & 1.1 & -1.0 & 0.47 & $-11.1_{-9.9}^{+8.8}$ & 20.81 & 20.4 \\
\hline
\end{tabular}

Table 2. Spectroscopic measurements for individual stars with either $\mathrm{S} / \mathrm{N}>3$ or radial velocity error less than $10 \mathrm{~km} \mathrm{~s}{ }^{-1}$ in the Hydrus 1 field. We include the internal ID of each star, right ascension, declination, signal to noise of the spectra, heliocentric radial velocity, effective temperature, surface gravity together with their uncertainties. The log-odds ratios of being a Hydrus 1 member star vs being a foreground contaminant from the chemo-dynamical model described in Sec. 4.3 and the $g, r$-band photometry from DECam observations are also included. Only a subset of stars is shown in the printed version of the table, the full version of the table is available online.

troscopic results. Only stars with RV uncertainty smaller than $10 \mathrm{~km} \mathrm{~s}^{-1}$ are shown. The third panel of the Figure shows the heliocentric velocity of observed stars vs distance from Hydrus's center, and the fourth panel shows line-ofsight velocity vs spectroscopic metallicity. The fifth panel shows the 1-D distribution of velocities. These panels clearly confirm that Hydrus 1 is a real stellar system. A large concentration of stars belonging to Hyi 1 is prominent at heliocentric velocities of $\sim 80 \mathrm{~km} \mathrm{~s}^{-1}$ and low metallicities $[\mathrm{Fe} / \mathrm{H}]<-2$. This group of stars is kinematically cold and is noticeably more metal-poor than other stars in the field that likely belong to the halo and/or MW disk. In total our sample seems to contain around 30 Hydrus 1 member stars. In the three rightmost panels of the Figure we also highlight the stars that are confidently classified as giants/sub-giants, i.e. $\log g<4, \sigma_{\log g}<1$ (shown in black rather than grey). This particular subsample of stars offers a particularly clear view of the Hydrus $1 \mathrm{RV}$ peak at $80 \mathrm{~km} \mathrm{~s}^{-1}$, with little contamination at different heliocentric velocities. This is expected given that stars belonging to Hydrus 1 should be mostly giant branch or horizontal branch stars and the contamination should mostly consist of thick disk dwarf stars and, to a much lesser extent, stellar halo giant stars at distances of $30 \mathrm{kpc}$.

\subsection{Chemo-Dynamical Modeling}

As a next step we proceed to modeling of the observed kinematic and chemical properties of Hyi 1 stars. To describe the distribution of velocities and chemical abundances in the data, we adopt a standard mixture model with different components representing Hyi 1, MW halo and disk. We also utilise the fact that the sample of stars classified as giants is much cleaner and should not be contaminated by thin and/or thick disk stars. Therefore we split our star sample into two subsamples: stars confidently classified as giants $\left(\log g<4, \sigma_{\log g}<1\right)$ and dwarfs (the rest).

A brief summary of the model is given below, where we provide the distribution of velocity $v$ and metallicity $[\mathrm{Fe} / \mathrm{H}]$ conditional on the object type $T$, a latent categorical variable describing whether a given star is a halo star $(T=\mathbf{h})$, disk star $(T=\mathbf{d})$ or Hydrus 1 (object) star $(T=\mathbf{o})$. For all the components: halo, disk and Hydrus 1 we assume that
$[\mathrm{Fe} / \mathrm{H}]$ abundances and velocities are independently Gaussian distributed. We also assume that radial velocity of Hydrus 1 stars could have a linear gradient on the sky, parameterised by two slopes $S_{\alpha}$ and $S_{\delta}$ along right ascension and declination respectively.

$$
\begin{aligned}
v \mid T=\mathbf{o}, \alpha, \delta & \sim \mathcal{N}\left(V_{o}+\right. \\
S_{\alpha}\left(\alpha-\alpha_{o}\right) \cos \delta_{0} & \left.+S_{\delta}\left(\delta-\delta_{o}\right), \sigma_{o}\right) \\
v \mid T=\mathbf{h} & \sim \mathcal{N}\left(V_{h}, \sigma_{h}\right) \\
v \mid T=\mathbf{d} & \sim \mathcal{N}\left(V_{d}, \sigma_{d}\right) \\
{[F e / H] \mid T=\mathbf{o} } & \sim \mathcal{N}\left([\mathrm{Fe} / H]_{o}, \sigma_{[\mathrm{Fe} / H], o}\right) \\
{[F e / H] \mid T=\mathbf{h} } & \sim \mathcal{N}\left([\mathrm{Fe} / H]_{h}, \sigma_{[\mathrm{Fe} / H], h}\right) \\
{[\mathrm{Fe} / H] \mid T=\mathbf{d} } & \sim \mathcal{N}\left([\mathrm{Fe} / H]_{d}, \sigma_{[\mathrm{Fe} / H], d}\right)
\end{aligned}
$$

Here the parameters $V_{h}, V_{d}, V_{o}, \sigma_{h}, \sigma_{d}, \sigma_{o}$ refer to the mean velocity and velocity dispersions of the halo, disk and Hydrus 1 populations respectively, while $[\mathrm{Fe} / \mathrm{H}]_{h},[\mathrm{Fe} / \mathrm{H}]_{d}$, $[\mathrm{Fe} / \mathrm{H}]_{o}, \sigma_{[\mathrm{Fe} / \mathrm{H}], h}, \sigma_{[\mathrm{Fe} / \mathrm{H}], d}, \sigma_{[\mathrm{Fe} / \mathrm{H}], o}$ refer to the means and dispersions in metallicity for the halo, disk and $\mathrm{Hy}-$ drus 1 .

The last part of the model describes the distribution over types $T$ for the samples of giants and dwarfs, conditional on angular distance bin from Hydrus 1's center $\left(D_{b i n}\right.$; we use 5 distance bins). We assume that the sample of giants has zero contamination from the MW disk and that the probability of observing a Hydrus 1 member is a monotonically declining function of distance. This reflects prior knowledge that the stellar density of Hyi 1 member stars with respect to contamination decreases with radius, without assuming a specific density law. This approach is preferred due to the complexity of taking into account the fiber allocation efficiency (this is similar to the non-parametric approach adopted in the EM-algorithm by Walker et al. (2009a)).

$$
\begin{aligned}
T \mid D_{\text {bin }}=k, \text { giant } \sim & \operatorname{Cat}\left(\left(f_{o, g, k}, 1-f_{o, g, k}, 0\right)\right) \\
T \mid D_{\text {bin }}=k, \text { dwarf } \sim & \operatorname{Cat}\left(\left(f_{o, w, k},\right.\right. \\
& \left.\left.\left(1-f_{o, w, k}\right) f_{h, d},\left(1-f_{o, w, k}\right) f_{d, w}\right)\right)
\end{aligned}
$$

The $\mathrm{g}$ and $\mathrm{w}$ subscripts in the parameters of the model refer to giant and dwarf samples respectively. For example $f_{o, g, k}$ refers to the probability of observing a Hydrus 1 star 

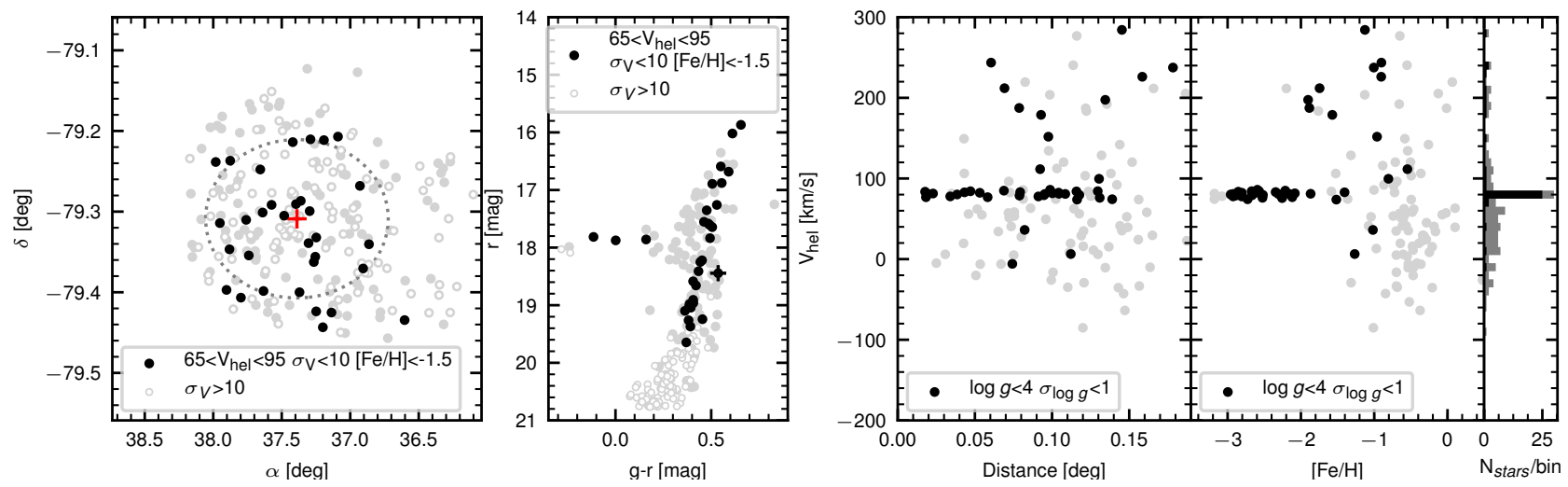

Figure 5. Left panel: Spatial distribution of spectroscopically targeted stars. The center of Hyi 1 is shown a by red cross. Darker circles indicate likely members, based on velocity and metallicity, while stars without a good velocity measurement are shown by empty gray circles. Filled grey circles show all other stars. The dotted line shows the half-light ellipse. Second panel: Color-magnitude diagram of spectroscopically targeted members. The meaning of the symbols is the same as in the left panel. We note that several horizontal branch stars are marked as likely members; however, a few stars at the very edge of the horizontal branch avoid this distinction due to high velocity uncertainties caused by lack of lines. A cross-hair marker indicates the carbon-enhanced metal poor star (Star 75) that is a member of Hydrus 1. Third panel: Heliocentric line-of-sight velocity vs angular distance from Hyi 1's center, for all stars with good velocity measurements (errors $\sigma_{v}<10 \mathrm{~km} \mathrm{~s}^{-1}$ ). Stars classified confidently as giants according to their spectra are shown by darker markers. Fourth panel: Heliocentric velocity vs spectroscopic metallicity for stars with good velocity measurements. The symbols used are the same as in the previous panel. Rightmost panel: Velocity histogram for the giant-only subsample (black) and all the stars with good velocity measurements (grey).

in the sample of giants in $\mathrm{k}$-th distance bin and $f_{d, w}$ refers to the probability of observing a disk star vs halo star in a sample of dwarfs (we assume that this probability is not dependent on the distance bin).

The model described above assumes error-free velocities and abundances; however, since all our measurements have non-negligible errors, we introduce these uncertainties into the model by assuming that all observed velocities and metallicities are normally distributed around their true values: $v_{\text {obs }} \sim \mathcal{N}\left(v, \sigma_{v, o b s}\right)$ and $[\mathrm{Fe} / \mathrm{H}]_{o b s} \sim \mathcal{N}\left([\mathrm{Fe} / \mathrm{H}], \sigma_{[\mathrm{Fe} / \mathrm{H}], o b s}\right)$ where $v_{o b s},[\mathrm{Fe} / \mathrm{H}]_{o b s}$ are the observed velocities and abundances, $\sigma_{v, o b s}$, $\sigma_{[\mathrm{Fe} / \mathrm{H}], o b s}$ are the observational uncertainties and $v$ and $[\mathrm{Fe} / \mathrm{H}]$ are true error-free parameters of each star.

The priors on the parameters of the model are mostly uninformative, $V_{h} \sim U(-300,300), V_{d} \sim U(-300,300)$, $V_{o} \sim U(60,100), \sigma_{h} \sim U(50,300), \sigma_{d} \sim U(10,300)$, $\sigma_{o} \sim U(0,15),[\mathrm{Fe} / \mathrm{H}]_{h} \sim U(-5,0),[\mathrm{Fe} / \mathrm{H}]_{d} \sim U(-1,0)$, $[\mathrm{Fe} / \mathrm{H}]_{o} \sim U(-5,0), \sigma_{[\mathrm{Fe} / \mathrm{H}], h} \sim U(0,2), \sigma_{[\mathrm{Fe} / \mathrm{H}], d} \sim$ $U(0,1), \sigma_{[\mathrm{Fe} / \mathrm{H}], o} \sim U(0,2), S_{\alpha} \sim U(-50,50), S_{\delta} \sim$ $U(-50,50)$, and $U(0,1)$ on all object type fractions $f_{d, w}$, $f_{o, g, k}, f_{o, w, k}$.

This chemo-dynamical model is implemented in the probabilistic language STAN (Carpenter et al. 2017) and is available in the supplementary materials for this paper. We run the model on all stars with velocity uncertainties less than $10 \mathrm{~km} \mathrm{~s}^{-1}$ (136 in total). We sampled the posterior using a Hamiltonian Monte-Carlo technique (Neal 2012) and No-U-Turn-Sampler (Hoffman \& Gelman 2011). After running 24 parallel chains for 10000 iterations each, we ensured that the resulting chains are in convergence using the Gelman-Rubin diagnostic (Gelman \& Rubin 1992) for each parameter as well as inspecting the marginal 2-D and 1-D posteriors, all of which show a single, smooth, well-behaved peak. To check that our model provides a good description of the data, we created replicated datasets from our best-fit
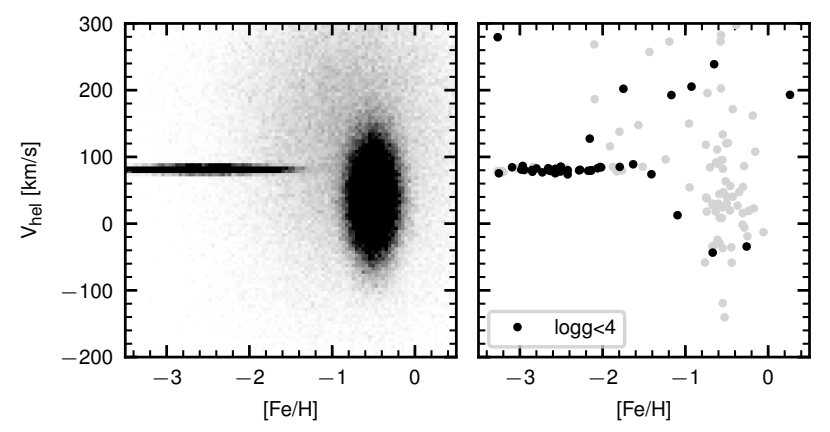

Figure 6. The demonstration of our chemo-dynamical model. Left panel: The 2-D density of stars in metallicity and radial velocity space showing our 3-component Gaussian mixture model. Right panel: The mock sample of 136 stars from our model, including both the giants $\log g<4, \sigma_{\log g}<1$ (black points) and dwarfs (grey points). The figure is meant to be very similar to the fourth panel of Figure 5. The observational uncertainties on both abundances and velocities have not been added to the simulated data points.

model. These are shown in Figure 6. The left panel of the Figure shows the overall smooth density in metallicity vs velocity space, as described by our model (assuming the same ratio between giant and dwarf subsamples as in our data), while the right panel shows an exact simulated replica of our dataset with 136 stars. The replication seems to resemble the actual dataset shown in Figure 5, providing some assurance that our model provides an adequate description of the data.

The numerical results of the chemo-dynamical model fit, i.e. the values of parameters and their uncertainties relevant for the Hydrus 1 system, are provided in Table 1, while 


\begin{tabular}{ccc}
\hline Parameter & Value & Unit \\
\hline$V_{\text {halo }}$ & $142 \pm 22$ & $\mathrm{~km} \mathrm{~s}^{-1}$ \\
$\sigma_{V, \text { halo }}$ & $134_{-14}^{+17}$ & $\mathrm{~km} \mathrm{~s}^{-1}$ \\
{$[\mathrm{Fe} / \mathrm{H}]_{\text {halo }}$} & $-0.83 \pm 0.13$ & $\mathrm{dex}$ \\
$\sigma_{[\mathrm{Fe} / \mathrm{H}] \text {,halo }}$ & $0.74 \pm 0.1$ & $\mathrm{dex}$ \\
$V_{\text {disk }}$ & $38.2 \pm 7.6$ & $\mathrm{~km} \mathrm{~s}^{-1}$ \\
$\sigma_{V, \text { disk }}$ & $49.5_{-5.7}^{+6.7}$ & $\mathrm{~km} \mathrm{~s}^{-1}$ \\
{$[\mathrm{Fe} / \mathrm{H}]_{\text {disk }}$} & $-0.52 \pm 0.03$ & $\operatorname{dex}$ \\
$\sigma_{[\mathrm{Fe} / \mathrm{H}], \text { disk }}$ & $0.17 \pm 0.04$ & $\operatorname{dex}$ \\
\hline
\end{tabular}

Table 3. Measurements of the disk and halo parameters from the chemo-dynamical model

Table 3 lists the parameter values for the contaminant components (disk and halo). Another output from our model is the membership probability for each star. We express these probabilities the form of log-odds of being a member star $\log \left(P_{\text {memb }} / P_{\text {nonmber }}\right)$ and we include them as a column in Table 2 . The uncertainties in those probabilities are obtained after marginalizing over all model parameters. We stress that membership probabilities for individual stars are often highly uncertain and model dependent; therefore, outputting just a single value of membership probability without uncertainty as it is common in the literature is meaningful only if model parameters (such as velocity dispersion or systemic velocity) are known precisely (which is almost never true).

Now we briefly summarize the main measurements from the chemo-dynamical model, with more detailed discussion in Section 5. The systemic heliocentric line-of-sight velocity of Hydrus 1 is $80.4 \pm 0.6 \mathrm{~km} \mathrm{~s}^{-1}$, which corresponds to a Galactic Standard of Rest velocity of $-94.1 \mathrm{~km} \mathrm{~s}^{-1}$. Given that the angle between the line of sight of Hydrus 1 and the line connecting the Galactic center to Hydrus 1 is only 17 degrees it is likely that the satellite is now on its way toward perigalacticon. The other key parameter in the chemodynamical model is the velocity dispersion of Hyi 1 , which is resolved and measured with high accuracy: $\sigma_{v}=2.69_{-0.43}^{+0.51}$ $\mathrm{km} \mathrm{s}^{-1}$. Assuming that the observed stellar velocities cleanly trace Hyi 1's gravitational potential, the total dynamical mass within the projected half-light radius of Hyi 1 can then be estimated using the estimator of Walker et al. (2009b) $M\left(<R_{1 / 2}\right) \sim 2.2 \times 10^{5} M_{\odot}$. This value corresponds to a mass-to-light ratio of $66_{-20}^{+29}$ solar units. Since mass-to-light ratio uncertainties are heavily asymmetric, we also compute the logarithm $\log _{10} M / L=1.82 \pm 0.16$, for which the uncertainties are close to Gaussian. As this ratio is clearly higher than that of the stellar population alone, this confirms suspcions based on morphology and structural parameters: Hydrus 1 is a dwarf galaxy dominated by dark matter.

The mean metallicity and metallicity dispersion of Hyi 1 are $[\mathrm{Fe} / \mathrm{H}]=-2.52 \pm 0.09$ and $0.41 \pm 0.08$, respectively. The average metallicity is consistent with the metallicity of other ultra-faint dwarfs and agrees with the stellarmass/metallicity relation traced by other dwarf galaxies (Kirby et al. 2013). We also note that the scatter in metallicities within Hydrus 1 is resolved and is significantly larger than zero, which is another indicator of the dwarf galaxy nature of the object (see e.g. Willman \& Strader 2012).

The best fit values for the line-of-sight velocity gradient in Hydrus 1 are $S_{\alpha}=\frac{d V_{h e l}}{d \alpha \cos \delta_{c e n}}=24.4 \pm 9.6 \mathrm{~km} \mathrm{~s}^{-1} \mathrm{deg}^{-1}$,

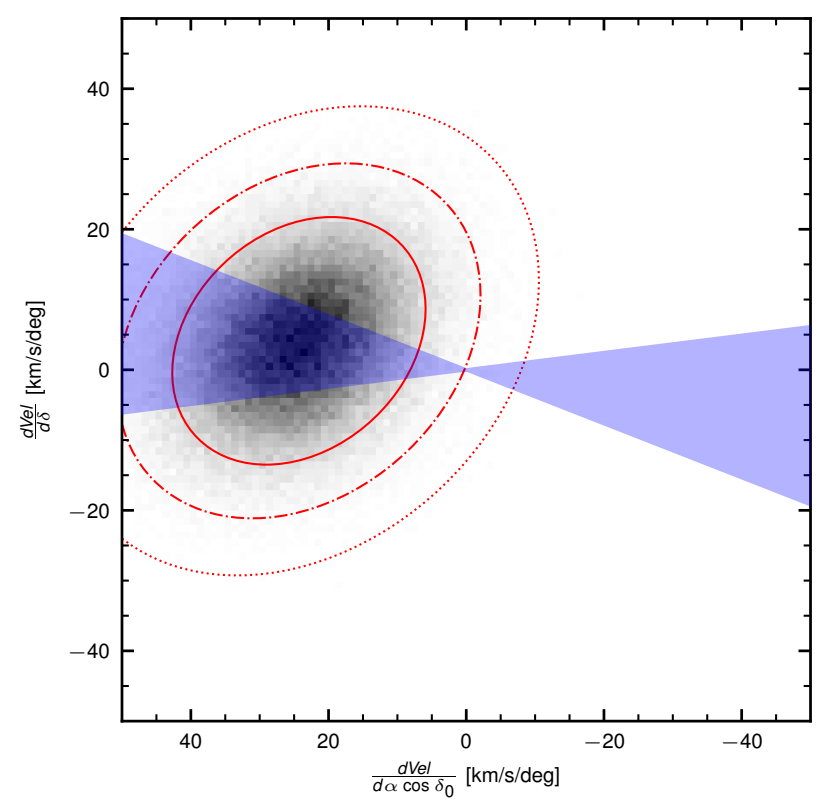

Figure 7. 2-D posterior density of the spatial line-of-sight velocity gradient as inferred from the M2FS kinematic data. The ellipses represent the posterior volume corresponding to 1,2 and $3 \sigma$. The blue wedge shows the range of direction of possible velocity gradients if they were aligned along the major axis of Hydrus 1. The width of the wedge reflects the uncertainty on the measurement of the positional angle of Hyi 1's major axis.

and $S_{\beta}=\frac{d V_{h e l}}{d \delta}=4.1 \pm 9.2 \mathrm{~km} \mathrm{~s}^{-1} \mathrm{deg}^{-1}$. We notice that the gradient along right ascension is marginally different from zero (at $\sim 2.5$ sigma significance level). To investigate this further, we show the $2-\mathrm{D}$ posterior on the velocity gradient in Figure 7 . We also show there the wedge that would correspond to the radial velocity gradient along the major axis of the system (the width of the wedge represents our uncertainty on the position angle of the major axis). We note that the marginal velocity gradient signal seems to be aligned with the major axis of Hydrus 1 . We discuss the implications of this result in more detail in Section 5.1.

\subsection{Chemical abundances}

As shown in the previous section, our spectroscopic dataset contains around 30 members of Hydrus 1, many of which are quite bright $(r<18)$ and have high-quality spectra. Therefore we synthesised spectra of 27 high signal-to-noise $(\mathrm{S} / \mathrm{N}>5)$ and high probability $\left(P_{H y i 1} / P_{b g}>1\right)$ Hydrus 1 stars in order to estimate their detailed chemical abundances. The observed wavelength region includes transitions of $\mathrm{Mg}$ (the $\mathrm{Mg}$ Ib triplet), $\mathrm{C}$ (the $\mathrm{C}_{2}$ Swan band), $\mathrm{Fe}$, and to a lesser extent, $\mathrm{Ti}$ and $\mathrm{Ni}$. We used the stellar parameters $T_{\text {eff }}$, $\log g$, and $[\mathrm{Fe} / \mathrm{H}]$ estimated from the previous Section, with microturbulence $v_{t}$ estimated using the empirical relationship from Kirby et al. (2008). We used the Castelli \& Kurucz (2004) plane-parallel model photospheres, and compiled a line list with atomic transitions from the Gaia-ESO Survey (Heiter et al. 2015) and molecular transitions (primarily $\mathrm{C}_{2}$ ) 
from Ram et al. $(2014)^{7}$. We used the 2014 July version of the MOOG spectral synthesis program (Sneden 1973).

We masked regions with stellar absorption lines and used a spline function to continuum-normalise the observations (Casey 2014). Continuum normalisation was relatively straightforward for all but one star, Star 75, which we found to be strongly enhanced in carbon, producing significant absorption at wavelengths bluer than $5165 \AA$. Carbon abundances could only be estimated for Star 75 , where we adopted a ${ }^{12} \mathrm{C} /{ }^{13} \mathrm{C}$ ratio of 3.5 , and we report upper limits on $[\mathrm{C} / \mathrm{H}]$ for most other members. Despite using a $\mathrm{C}_{2}$ molecular line list that was compiled specifically for accurate predictions of carbon from the Swan $\mathrm{C}_{2}$ bands, we found that there were significant discrepancies between the predicted and observed wavelengths of the $\mathrm{C}_{2}$ feature near $5165 \AA$ in Star 75 . Although the $\mathrm{S} / \mathrm{N}$ in this spectrum is relatively low $\left(S / N \approx 6\right.$ pixel $\left.^{-1}\right)$, the predicted 'saw-tooth' spectral features seem to almost grow out of phase with the observations (Figure 8), even after allowing for a small residual radial velocity shift to fit the observations. Adopting different isotopic fractions of ${ }^{12} \mathrm{C} /{ }^{13} \mathrm{C}$ did not resolve the issue. Discrepancies in wavelength position of this bandhead have been noted in other works (Brooke et al. 2013; Ram et al. 2014), but not to the extent that seems apparent in Star 75 . On the other hand, the low $\mathrm{S} / \mathrm{N}$ ratio does make it difficult to properly quantify the extent of the wavelength discrepancies. The estimated abundance ratio of $[\mathrm{C} / \mathrm{Fe}]=+3$ seems to be largely independent of these wavelength offsets, because if we convolve the observations and data with a Gaussian kernel to smooth out the spectral features and produce a near-continuous opacity blueward of $5165 \AA$ that is due to the $\mathrm{C}_{2}$ Swan band, the model and observations fit excellently with $[\mathrm{C} / \mathrm{Fe}]=+3$. The molecular features, and the level of near-continuous opacity when smoothed, is extremely sensitive to the level of $[\mathrm{C} / \mathrm{H}]$, so the estimated abundance ratio of $[\mathrm{C} / \mathrm{H}]$ seems to be largely insensitive to the differences in the wavelengths of the $\mathrm{C}_{2}$ bandhead.

We were able to estimate $[\mathrm{Mg} / \mathrm{H}]$ abundances for all Hydrus 1 members. The Ti and Ni atomic lines in this wavelength region are relatively weak, only allowing for one measurement of $[\mathrm{Ni} / \mathrm{H}]=-2.15 \pm 0.11$ for Star 154 , and upper limits of $[\mathrm{Ni} / \mathrm{H}]<-2.68$ for Star 165 , and $[\mathrm{Ti} / \mathrm{H}]<-2.46$ for Star 52. Although these limits are not very informative, we include them here for completeness. We were able to estimate $\mathrm{Fe}$ abundances by spectral synthesis for most stars, except for those with very low $\mathrm{S} / \mathrm{N}$ ratios (e.g., $\mathrm{S} / \mathrm{N}$ $\approx 5$ pixel $\left.^{-1}\right)$ or very hot stars $\left(T_{\text {eff }} \approx 8000 \mathrm{~K}\right)$. These estimates are in very good agreement with those estimated by template fitting in Section 4.2: well within the estimated uncertainties of either method. Uncertainties in chemical abundances were estimated during the fitting process by projecting the derivatives in the synthesised spectra with the uncertainties in the continuum-normalised fluxes. The estimated abundances, uncertainties, and upper limits, are given in Table 4 . We discuss these measurements in the next section of the paper.

\footnotetext{
7 Available in MOOG format from http://www.as.utexas.edu/ chris/lab.html
}

\begin{tabular}{lccc}
\hline Star ID & {$[\mathrm{C} / \mathrm{H}]$} & {$[\mathrm{Mg} / \mathrm{H}]$} & {$[\mathrm{Fe} / \mathrm{H}]$} \\
\hline 4 & $<-0.32$ & $-2.64 \pm 0.11$ & $-2.42 \pm 0.10$ \\
26 & $<-0.15$ & $-3.04 \pm 0.15$ & $-2.15 \pm 0.10$ \\
30 & $<-0.74$ & $-2.27 \pm 0.10$ & $-1.74 \pm 0.10$ \\
34 & $<-0.64$ & $-2.72 \pm 0.12$ & $-2.94 \pm 0.10$ \\
49 & $<-0.82$ & $-2.38 \pm 0.10$ & $-2.97 \pm 0.10$ \\
52 & $<-1.13$ & $-1.78 \pm 0.10$ & $-2.38 \pm 0.10$ \\
61 & $<-0.43$ & $-2.07 \pm 0.16$ & -3.08 \\
68 & $<-1.03$ & $-2.40 \pm 0.10$ & $-2.67 \pm 0.10$ \\
69 & $<-0.71$ & $-2.26 \pm 0.11$ & $-2.61 \pm 0.10$ \\
72 & $<-0.74$ & $-2.53 \pm 0.11$ & $-2.80 \pm 0.14$ \\
75 & $-0.18 \pm 0.20$ & $-2.77 \pm 0.14$ & -3.18 \\
77 & $<-1.06$ & $-1.01 \pm 0.10$ & $-1.60 \pm 0.10$ \\
102 & $<0.98$ & $-2.61 \pm 0.13$ & $-3.17 \pm 0.18$ \\
119 & $<-0.47$ & $-2.43 \pm 0.12$ & $-2.87 \pm 0.10$ \\
139 & & $-2.13 \pm 0.10$ & -2.26 \\
149 & $<1.82$ & $-0.70 \pm 0.12$ & -1.40 \\
150 & $<-1.28$ & $-2.33 \pm 0.10$ & $-2.52 \pm 0.10$ \\
151 & & $-2.28 \pm 0.34$ & -2.31 \\
154 & $<-1.43$ & $-2.34 \pm 0.10$ & $-2.55 \pm 0.10$ \\
165 & $<-1.58$ & $-2.55 \pm 0.10$ & $-2.85 \pm 0.10$ \\
182 & $<-1.01$ & $-2.57 \pm 0.10$ & $-3.01 \pm 0.10$ \\
188 & & $-2.26 \pm 0.29$ & $-2.23 \pm 0.11$ \\
209 & $<-0.12$ & $-2.36 \pm 0.14$ & $-3.12 \pm 0.13$ \\
221 & & $-1.84 \pm 0.10$ & -2.21 \\
222 & $<0.07$ & $-2.24 \pm 0.23$ & $-2.68 \pm 0.12$ \\
225 & $<-0.71$ & $-2.59 \pm 0.10$ & $-2.54 \pm 0.10$ \\
\hline & & &
\end{tabular}

Table 4. Chemical abundances and upper limits estimated via spectral synthesis (Section 4.4) for high probability members of Hyi 1.

\section{DISCUSSION}

In this section we discuss and summarize the key results from the photometric, chemical and dynamical analyses of Hydrus 1 presented in Sections 3 and 4. In particular we focus on possible radial velocity gradient of the dwarf, its dark matter content, chemical properties, connection to the Magellanic Clouds and dark matter annihilation searches.

\subsection{Radial velocity gradient}

The radial velocity gradient that we detect in Hyi 1 is only marginally significant, at $2.5 \sigma$, and seems to be aligned with the major axis of the system. If we assume that it is not a statistical fluctuation, possible causes of this gradient would include satellite rotation, perspective rotation and tidal disruption. Perspective rotation (Kaplinghat \& Strigari 2008; Walker et al. 2008) is caused by projection effects from tangential motion of an object with large angular size. However as Hyi 1's size is only $\sim 0.1^{\circ}$ on the sky, the observed gradient of $\sim 24 \mathrm{~km} \mathrm{~s}^{-1} \mathrm{deg}^{-1}$ would imply an unrealistically high tangential velocity $\left(\gtrsim 1000 \mathrm{~km} \mathrm{~s}^{-1}\right)$, and is therefore an unlikely cause of the observed gradient. Another possible explanation is tidal disruption of the satellite. This has been claimed to be observed in a few MW satellites (Adén et al. 2009; Collins et al. 2017), albeit at low levels of significance. We note however that the observed gradient is much larger than expected from a simple velocity gradient along the orbit in the MW gravitational potential. Therefore, in order to explain the gradient in terms of disruption, significant 


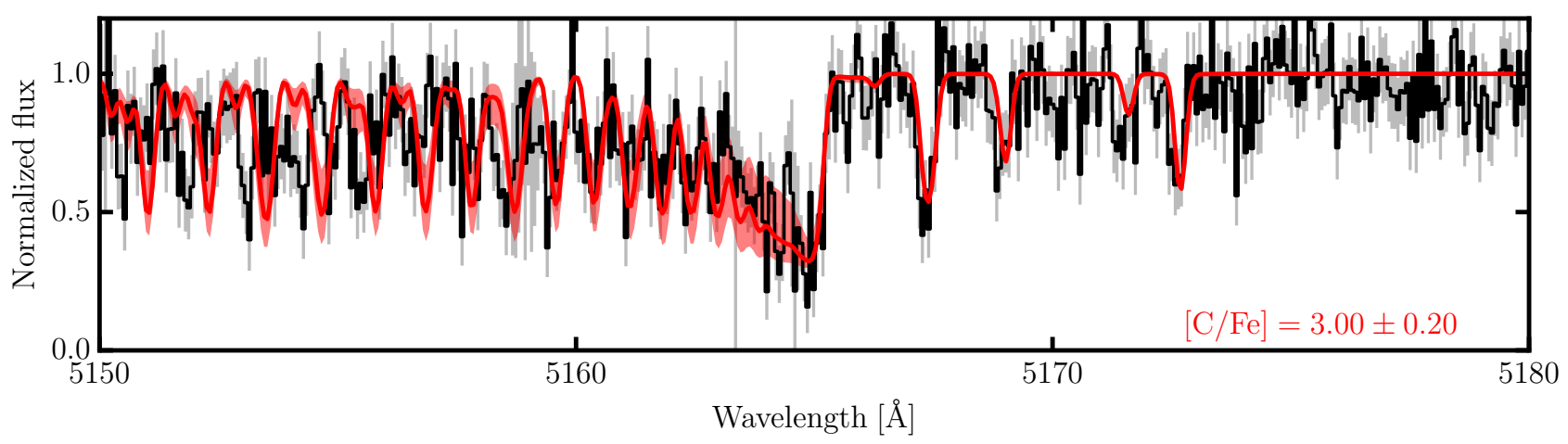

Figure 8. Spectrum of the carbon enhanced extremely metal-poor (CEMP) Hydrus 1 star (star 75) with spectral synthesis model overplotted in red. Gray shading shows the observational uncertainties in the measured spectrum and red shading shows the uncertainties associated with the spectral synthesis parameters. The spectrum shows a strong carbon absorption band and low metallicity indicative of CEMP stars.

fine-tuning of the orbit would be required (Küpper et al. 2010; Martin \& Jin 2010; Küpper et al. 2017). Furthermore, the low ellipticity of Hyi 1 and the apparent regularity of the satellite and its density profile argue against this possibility. Finally, the velocity gradient in the system could be explained by satellite rotation. Assuming the measured radial velocity gradient along the major axis corresponds to solid-body rotation, we estimate a rotation velocity of $3 \mathrm{~km} \mathrm{~s}^{-1}$ at the half-light radius, which is comparable to the velocity dispersion of the system. To illustrate the plausibility of this interpretation, we show in Figure 9 the radial velocity of possible Hydrus 1 members (selected based on metallicities only) as a function of position angle of the system, with the curve showing the line-of-sight velocity model evaluated at the half-light radius (essentially interpreted as satellite rotation). We note that the model seems to fit the data well, matching the observed RV changes as a function of positional angle. If the rotation hypothesis is a correct interpretation of the data, that would make Hydrus 1 the first ultra-faint dwarf with significant rotation. Why would be Hydrus 1 be an exception? It is difficult to say for sure, but it is possible that at least some of the dwarf satellites of the MW had disk-like morphology before accretion, and were then transformed into spheroidal galaxies by a process known as tidal stirring (Mayer et al. 2001; Kazantzidis et al. 2011). In this case some of the satellites could still be in the transformation phase with significant rotational support. That would mean that Hydrus 1 became a satellite very recently.

However, due to the low statistical significance of the rotation signal, we urge caution and emphasise the need for more data to confirm or refute it.

\subsection{Nature of Hydrus 1}

While the nature of Hydrus 1 as a dwarf galaxy is established by its size, dark matter content and internal metallicity distribution, in this Section we put Hyi 1 in context with other systems of similar structural and chemo-dynamical properties.

With a circularised effective radius of $\sim 50 \mathrm{pc}$ and lumi-

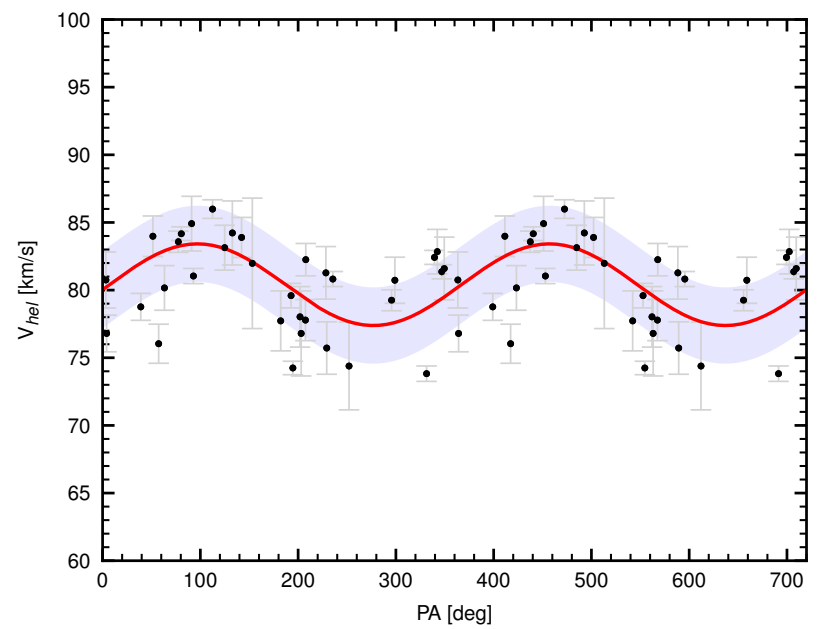

Figure 9. Line-of-sight velocities of Hydrus 1 stars vs position angle. Only stars with velocity uncertainty less than $5 \mathrm{~km} \mathrm{~s}^{-1}$ and $[\mathrm{Fe} / \mathrm{H}]<-1$ are shown. All the data points have been plotted twice, once with the original position angles, and once with position angles offset by 360 degrees. The red curve shows the radial velocity change according to the best fit velocity gradient evaluated at half-light radius (corresponding to $\sim 3 \mathrm{~km} \mathrm{~s}^{-1}$ rotation velocity). The shaded region around the curve has the half-width of the best fit velocity dispersion of the system.

nosity of $M_{V} \sim-4.7$, Hydrus 1 sits in an ambiguous between dwarf galaxies and extended globular clusters (GCs) in the luminosity-size space. Figure 10 shows all known MW satellites (dSphs and GCs) together with Hydrus 1. The objects similar in size and luminosity to Hydrus 1 are the Hydra II satellite (Martin et al. 2015) with a luminosity of $M_{V}=-4.8$ and size $r_{h}=68 \mathrm{pc}$, and the Sagittarius II satellite (Laevens et al. 2015) with a luminosity of $M_{V}=-5.2$ and half-light radius of $\sim 37 \mathrm{pc}$. The Crater 1 (or Laevens 1 ) satellite (Belokurov et al. 2014; Laevens et al. 2014) has similar luminosity $\left(M_{V}=-5.3\right)$ to Hydrus 1 , but significantly smaller size $(\sim 20 \mathrm{pc})$. The classification of Hydra II is somewhat unclear 


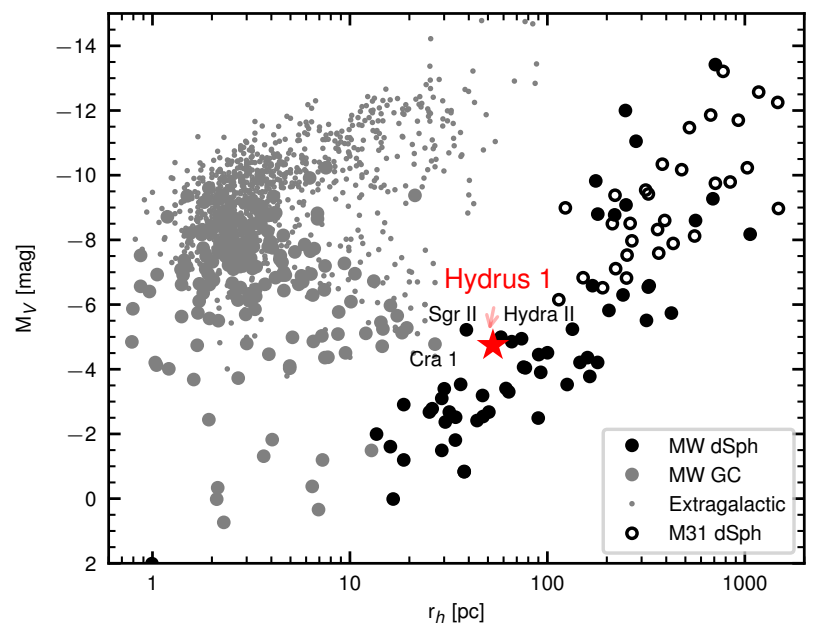

Figure 10. Distribution of sizes and luminosities of various faint stellar systems. Hydrus 1 is shown by a red star. MW/M31 dwarf spheroidal galaxies are shown by black filled/empty circles, respectively. Large grey circles show MW globular clusters, while small grey circles are showing extra-galactic compact stellar systems $(<100 \mathrm{pc})$ from Brodie et al. (2011). Data are taken from Figure 6 of Torrealba et al. (2018) and include the most recent dwarf galaxy and globular cluster discoveries.

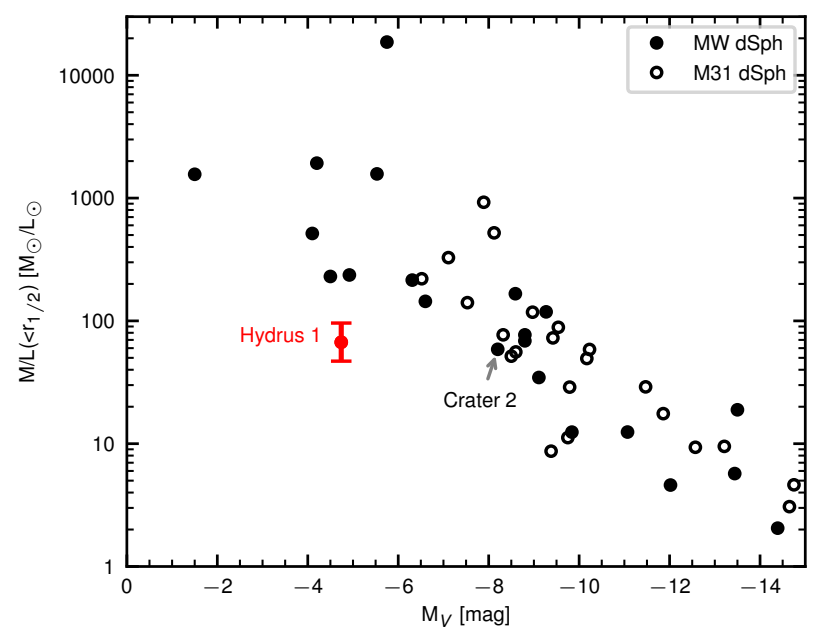

Figure 11. Dynamical mass-to-light ratio as a function of luminosity for dwarf satellites of the MW and M31. Hyi 1 is marked by a red circle. Data come from the 2015 version of the dwarf galaxy database by McConnachie (2012) as well as additional data by Caldwell et al. (2017); Li et al. (2018).

because its velocity dispersion is only marginally resolved; however, (Kirby et al. 2015) argue that its chemical abundances favor a dwarf galaxy interpretation. Sagittarius II does not have any velocity dispersion measurement. Finally, the Crater 1 satellite currently seems to be classified as an extended globular cluster based on the star-formation history and unresolved velocity dispersion (Weisz et al. 2016; Voggel et al. 2016). We note however that the mass-to-light ratio of Crater 1 from Voggel et al. (2016) $M / L=8.52_{-6.5}^{+28.0}$ is formally consistent with both the absence of dark matter and a significant amount of dark matter.

While the mass-to-light ratio measurement of $\log M / L=1.82 \pm 0.16$ in Hyi 1 unambiguously indicates the presence of dark matter, it is worth comparing Hyi 1 to other dwarfs of similar luminosity. Figure 11 shows mass-to-light ratio as a function of luminosity for MW and M31 satellite dwarf galaxies; Hydrus 1 is identified by a red marker. We note that Hyi 1 seems to be an outlier, possessing a mass-to-light ratio well below (by a factor of 3) other galaxies with similar luminosities. What makes Hydrus 1 such an outlier? One possibility is that some of the faint objects like Hydra II, Crater 1 and Hydrus 1 and others that occupy the area in size-luminosity space between globular clusters and dwarf galaxies could be intermediate-type objects with small but nonzero dark matter content and mass-to-light ratios smaller than other typical ultra-faint dwarfs. The origin of these systems could then be related either to tidal disruption after accretion (Peñarrubia et al. 2008) or to their initial formation (Ricotti et al. 2016). Testing this hypothesis would be quite difficult, however, as the known objects near the dwarf galaxy/globular cluster interface have small velocity dispersions and we lack high enough quality data on these systems to rule out a smooth transition from dark matter dominated objects to dark matter-free globular clusters.

Another possible explanation for the apparent low massto-light ratio of Hyi 1 could be related to our tentative measurement of the line-of-sight velocity gradient (see Section 4.3 and 5.1). If the gradient is real and is caused by galaxy rotation, the mass estimator based on velocity dispersion alone could be underestimating the dynamical mass and therefore the mass to light ratio. To gauge the possible impact of this we make a simple modification of the Walker et al. (2009b) estimator, adding a rotation term

$$
M\left(<r_{1 / 2}\right)=\frac{5}{2 G}\left(\sigma^{2}+\frac{2}{5} V_{\text {rot }}^{2}\right) r_{1 / 2}
$$

Assuming that the rotation velocity can be obtained from the radial velocity gradient evaluated at half light radius $V_{\text {rot }}=\frac{d V}{d \alpha \cos \delta_{\text {cen }}} r_{1 / 2}$ and that we observe the rotation in the edge-on orientation, we can obtain a half-light dynamical mass of $M\left(<r_{1 / 2}\right)=2.60_{-0.70}^{+0.98} 10^{5} \mathrm{M}_{\odot}$. This measurement is larger by $\sim 20 \%$ than the dynamical mass calculated without taking into account the rotation and would imply the mass-to-light ratio of $\sim 80$. This is not enough, however, to put Hydrus 1 in line with other ultra-faints on Fig. 11. Of course, the impact of rotation can be increased if we assume that the rotation plane is not viewed edge-on, in which case the true rotation would be higher by $\cos i$ and could in principle increase the dynamical mass of Hydrus 1 to match the overall relation in Fig. 11.

\subsection{Connection to the Magellanic Clouds}

As seen in Figure 1, the location of the Hydrus 1 satellite on the sky is right in between the Large and the Small Magellanic Clouds. Moreover, as discussed in Section 3.2, the satellite orientation on the sky appears to be aligned with the direction of the Clouds' motion on the sky. Thus, it is not imprudent to ask: how likely is the association of Hydrus 1 


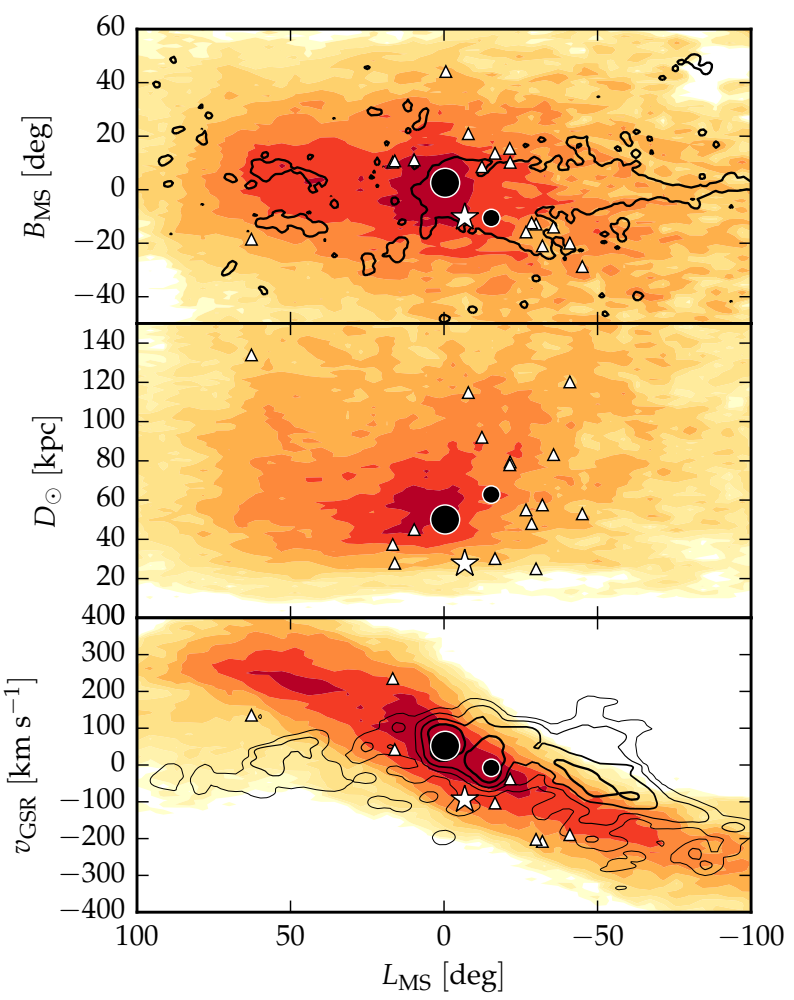

Figure 12. Density of the LMC debris in the maximumlikelihood model of Jethwa et al. (2016). Top: Probability distribution of the LMC satellites on the sky in the Magellanic coordinate system as defined by Nidever et al. (2008). Black contours show neutral hydrogen gas in the Magellanic Stream. Large and small black circles mark the locations of the LMC and the SMC respectively. White star marker shows the location of Hydrus 1, while white triangles show the location of known MW satellites. Middle: Colored density contours represent the expected probability distribution of the LMC satellites in the Magellanic latitude vs heliocentric distance space. The meaning of the symbols is the same as in the top panel. The contours for the Magellanic stream are not shown due to the lack of distance estimates available. Bottom: Same as above but for the galactocentric line-of-sight velocity vs Magellanic latitude.

with the Magellanic accretion event? In fact, the Magellanic hypothesis has recently been invoked to explain the nature of many of the satellites discovered in the vicinity of the Clouds in the past few years (Koposov et al. 2015a,b; DrlicaWagner et al. 2015b; Torrealba et al. 2018). Unfortunately, it is impossible to give the exact answer to this question without the complete set of the satellite's phase-space coordinates combined with the accurate mass estimates of the LMC and the SMC. Nonetheless, one may draw some useful conclusions by comparing the numerical simulations of the Magellanic Clouds' disruption to the observed properties of the satellite (see e.g. Nichols et al. 2011; Jethwa et al. 2016; Sales et al. 2017).

Figure 12 shows the expected distributions of the particles stripped from the Large Magellanic Cloud as computed by Jethwa et al. (2016). The top panel gives the positions on the sky in the Magellanic Stream coordinate sys- tem (see Nidever et al. 2008). The middle panel presents the dependence of the heliocentric distance on the Magellanic stream longitude $L_{\mathrm{MS}}$. Finally, the bottom row reveals how the galactocentric radial velocity evolves with $L_{\mathrm{MS}}$. Overplotted on top of the distribution of the simulated particles are the positions of the currently known MW satellites together with that of Hydrus 1. Unsurprisingly, as illustrated in the top panels, on the sky, Hyi 1 sits right at the peak of the predicted debris distribution. However, the match between the simulations and the observations appears to be much less convincing if the other two phase-space projections are considered. In particular, Hyi 1 is significantly closer than the majority of the LMC debris, yet its heliocentric distance is in marginal agreement with the maximum likelihood model prediction. Similarly, the line-of-sight velocity measurement of Hyi 1 is not well reproduced by the model, but appears compatible with the broad trend of increasing $V_{\mathrm{GSR}}$ as a function of $L_{\mathrm{MS}}$. It is difficult to speculate how informative the agreement between the observed position of Hydrus 1 and the Jethwa et al. (2016) models is, given that the width of the LOS velocity distribution is $\sim 200 \mathrm{~km} \mathrm{~s}^{-1}$. Naively, this would imply that there is a $\sim 50 \%$ chance that a random MW halo object, not related to the MC in-fall, will possess a velocity in agreement with the Jethwa et al. (2016) predictions.

Some of the thickness of the density contours of the LMC debris in Figure 12 may be due to the maximum likelihood model of Jethwa et al. (2016) attempting to explain the wide spread of the positions of the DES satellites on the sky and in the distances along the line of sight. While this model generates a broad cloud of tidal debris which envelopes most of the satellites, it appears to be incorrect in detail. As pointed out by Jethwa et al. (2016), the satellite distribution around the LMC is rather anisotropic, with many of the satellites forming a narrow sequence aligned with the projection of the SMC's orbital plane onto the sky (see Figure 7 of Torrealba et al. (2018)). Therefore, given that the above maximum likelihood model may be over-bloated, it is useful to conduct a different experiment and explore instead whether there exists any model of the Clouds' accretion in which the observed phase-space position of Hyi 1 is significantly more likely. Motivated by the recently reported measurements giving preference to the "light" Galaxy (see e.g. Gibbons et al. 2014; Williams et al. 2017; Eadie et al. 2017; Patel et al. 2018), we focus on a particular simulation, in which the MW/LMC/SMC masses are $75 / 5 / 4 \times 10^{10} M_{\odot}$. For this simulation, Figure 13 shows the density of the LMC particles in the plane spanned by the line-of-sight velocity $V_{\mathrm{GSR}}$ and the distance from the LMC, $R_{\mathrm{LMC}}$, marginalized over 150 different values of the Clouds' proper motions. Note that we select particles in a narrow slice of Magellanic longitudes corresponding to that of Hyi 1 and a range of Magellanic latitudes. The left panel of the Figure shows the view of the LMC debris not too dissimilar to the ML model predictions displayed in Figure 12. Here, a broad - albeit lumpy - $V_{\mathrm{GSR}}$ distribution is visible, without any strong trends as a function of $R_{\mathrm{LMC}}$. However, as the middle panel of Figure 13 reveals, there exists a noticeable dynamical age gradient across the ( $\left.V_{\mathrm{GSR}}, R_{\mathrm{LMC}}\right)$ plane. More precisely, the particles stripped recently, i.e. less than $0.1 \mathrm{Gyr}$ ago populate low $V_{\mathrm{GSR}}$ values across a wide range of the LMC distance. However, the debris unbound from the Cloud more than $0.1 \mathrm{Gyr}$ 

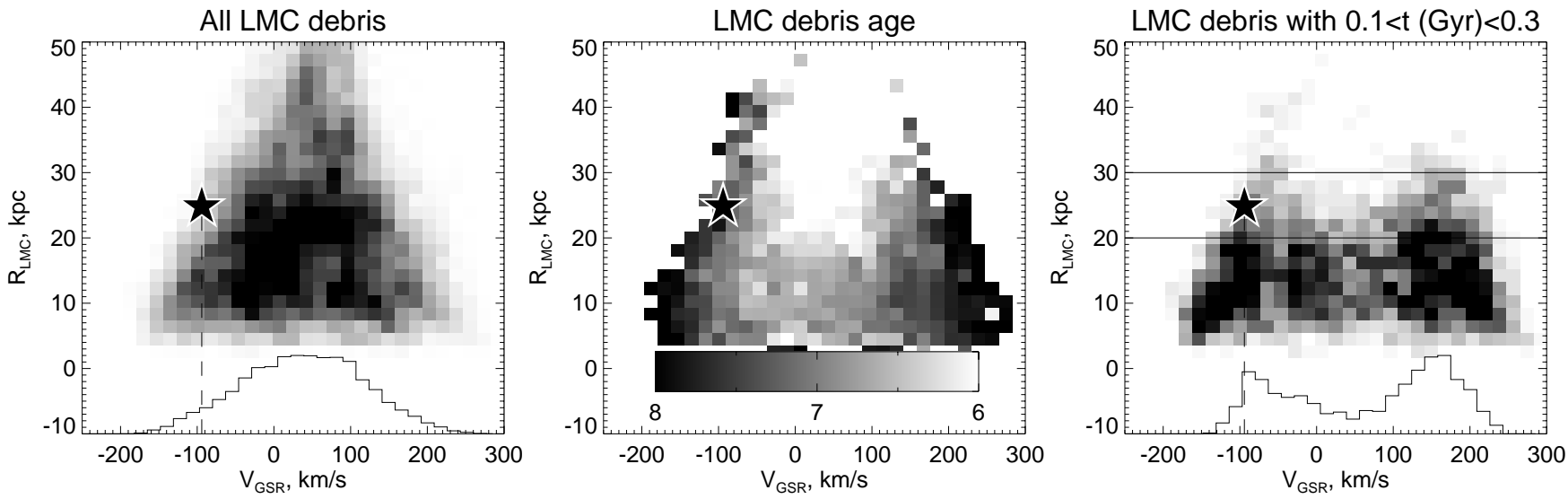

Figure 13. Distribution of the line-of-sight velocity $V_{\mathrm{GSR}}$ of the simulated LMC debris as a function of the distance from the Cloud, $R_{\mathrm{LMC}}$. In this particular simulation, the MW/LMC/SMC masses are fixed at $75 / 5 / 4 \times 10^{10} M_{\odot}$. We select particles at the same Magellanic longitude $L_{\mathrm{MS}} \sim-7^{\circ}$ as that of Hyi 1 , but in a range of latitudes $B_{\mathrm{MS}}$. The location of the Hyi 1 satellite is marked with a black star. Left: Density of particles in the $V_{\mathrm{GSR}}, R_{\mathrm{LMC}}$. The black histogram shows the $1 \mathrm{D}$ distribution of $V_{\mathrm{GSR}}$. Middle: Mean dynamical age of the particles as a function of $V_{\mathrm{GSR}}$ and $R_{\mathrm{LMC}}$. According to the greyscale bar displaying the logarithm of the stripping time in years, the bulk of the debris was removed from the LMC less than 0.5 Gyr ago. There is a clear age gradient as a function of $V_{\mathrm{GSR}}$, with older debris in the leading (trailing) tail possessing higher negative (positive) velocities. Right: Only debris stripped between 0.1 and 0.3 Gyr ago are shown. Histogram shows the $1 \mathrm{D} V_{\mathrm{GSR}}$ distribution for particles with $20<R_{\mathrm{LMC}} / 1 \mathrm{kpc}<30$, i.e. those at distances from the LMC similar to that of Hyi 1 . Note the strong bimodality of the velocity distribution.
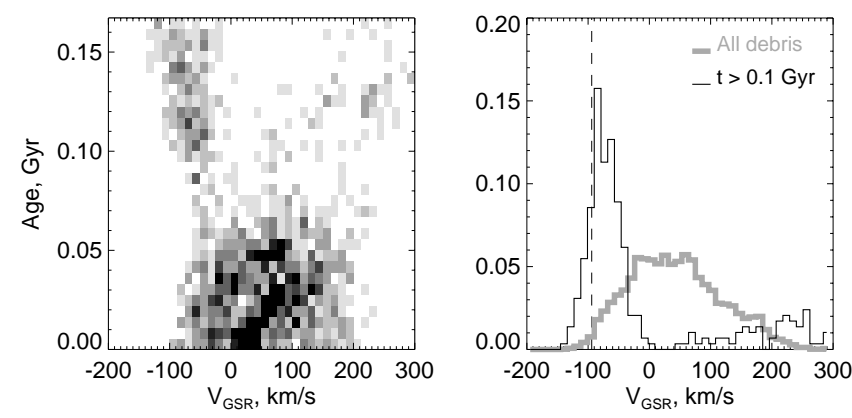

Figure 14. Distribution of the LMC debris in the simulation considered in Figure 13 within $10 \mathrm{kpc}$ of Hyi 1. Left: Density of particles in the plane of Galactocentric line-of-sight velocity and dynamical age (time of stripping from the LMC). Notice two distinct clouds of debris: the one corresponding to the most recently unbound particles, those with age $<0.1$ Gyr and a range of velocities, and the second group with $t>0.1$ Gyr. Right: $1 \mathrm{D}$ velocity distribution of all debris (debris with $t>0.1 \mathrm{Gyr}$ ) shown in grey (black). Dashed line marks the measured $V_{\mathrm{GSR}}$ of Hyi 1.

ago form tight sequences with high negative and high positive velocities, likely corresponding to the leading and the trailing tails. Hyi 1 (shown as a black star) can be found on the leading sequence, whose $V_{\mathrm{GSR}}$ velocity decreases away from the LMC. As displayed in the right panel of the Figure, if only dynamically old particles are selected, the histogram of their radial velocities becomes strongly bimodal with Hyi 1 situated near the peak of the leading debris distribution.

We confirm that the old debris, i.e. those stripped longer than 0.1 Gyr ago have radial velocities similar to that measured for Hyi 1 by selecting particles within $10 \mathrm{kpc}$ of the satellite (in 3D). The distribution of the simulated debirs is shown in Figure 14, where two clumps with distinct age properties are apparent (left panel). The dynamically old debris occupy very narrow velocity range very close to $V_{\mathrm{GSR}}$ of Hyi 1 (right panel of the Figure). It therefore appears that in the low-mass MW, some of the satellites stripped in the early phases of the LMC disruption can populate the leading debris tail (with kinematics similar to that measured for Hydrus) while staying in a close proximity to the Cloud. In $3 \mathrm{D}$, Hydrus 1 is only $24 \mathrm{kpc}$ away from the LMC. Interestingly, although this is nominally larger than the recent low bounds on the tidal radius of the Cloud (Mackey et al. 2016; Besla et al. 2016), it is nonetheless quite close to it.

The final answer as to the possibility of the association of Hyi 1 and the LMC will likely be procured using the data from the second data release of the Gaia satellite, which will provide plenty of the satellite's members with measured proper motion with individual uncertainties

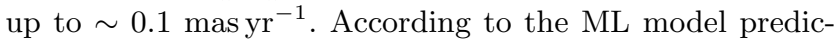
tion of Jethwa et al. (2016), a satellite stripped from the LMC and currently observed at the position of Hyi 1 with $V_{\mathrm{GSR}} \sim-95 \pm 30 \mathrm{kms}^{-1}$ would have $\mu_{\mathrm{W}}=-2.85 \pm 1.7$

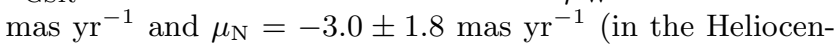
tric frame). On the other hand, if we select only the particles contributing to the overdensity of old debris around the location of Hyi 1 as shown in Figure 14, we obtain $\mu_{\mathrm{W}}=2.0 \pm 0.6$

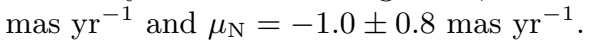

\subsection{Dark matter annihilation}

Hydrus 1's close proximity makes it an interesting target for indirect searches for photons that might be released as products of dark matter particle interactions - e.g., annihilation or decay. In recent years some of the deepest searches and strongest constraints on annihilating dark matter have come from gamma-ray observations of the Milky 
Way's dwarf spheroidal galaxies, and in particular from the ultrafaint population revealed by deep sky surveys (e.g. He et al. 2015; Geringer-Sameth et al. 2015a; Ackermann et al. 2015; Geringer-Sameth et al. 2015b; Drlica-Wagner et al. 2015a; Li et al. 2016; Albert et al. 2017). Here we quantify the expected signal from Hydrus 1 and perform a search for gamma-ray emission with data from the Fermi Gamma-ray Space Telescope.

For a given particle physics model (i.e., particle mass, interaction cross section), the flux of the expected photon signal depends on the distribution of dark matter within the source. For annihilation the flux is proportional to the $J$-profile:

$$
\frac{d J(\hat{n})}{d \Omega}=\int_{l=0}^{\infty}[\rho(l \hat{n})]^{2} d l,
$$

where $l$ is the line of sight distance in direction $\hat{n}, \rho(l \hat{n})$ is the dark matter density at location $l \hat{n}$ and $\Omega$ is solid angle. Following the procedure described in detail by Geringer-Sameth et al. (2015), we use the spherical Jeans equation to estimate the dark matter density profile, $\rho(r)$, of Hydrus 1 . We adopt the sample of 31 stars for which our measurements of position, velocity and metallicity imply membership probability $>0.95$. Assuming hydrostatic equilibrium, spherical symmetry, constant velocity anisotropy and negligible contamination by unresolved binary stars, we adopt the same fiveparameter dark matter halo model and priors as described by Geringer-Sameth et al. (2015). We also follow the same procedure to obtain the marginalized posterior probability distribution for $\log _{10}\left[J(\theta) /\left(\mathrm{GeV}^{2} \mathrm{~cm}^{-5}\right)\right]$, where $J(\theta)$ is the integral of $d J / d \Omega$ over a solid angle corresponding to angular radius $\theta$. In particular, since the kinematic data do not constrain the dark matter halo beyond the orbits of the star in the dataset, we truncate $\rho(r)$ at the radius of the outermost member star (see Geringer-Sameth et al. (2015, Sec. 6.4)). For Hydrus 1 the estimated (deprojected) distance to the outermost member star is $140 \mathrm{pc}\left(2.6 r_{\text {half }}\right)$, corresponding to a maximum angle $\theta_{\max }=0.29^{\circ}$, beyond which $d J / d \Omega$ is zero. This truncation gives a conservative estimate of the amplitude predicted for an annihilation signal under a given particle physics model.

The resulting measurement of Hydrus 1's $J$ value is $\log _{10} J\left(\theta_{\max }\right) /\left(\mathrm{GeV}^{2} \mathrm{~cm}^{-5}\right)=18.33_{-0.34}^{+0.38}$ (16th, 50th, and 84th percentiles of the posterior). In Fig. 15 we compare Hydrus 1 with Milky Way dwarfs in the "top tier" of $J$ values. For purposes of ranking it is important to treat the dwarfs as similarly as possible when computing $J$ 's. Different analyses of the same kinematic data set often yield $J$ values differing by amounts comparable to the statistical error. This is due to choices in the Jeans analysis (e.g. form of the dark matter profile, prior ranges, halo truncation, the assumption of spherical symmetry, etc.). In Fig. 15 all $J$ values represented by green diamonds are computed using the method (Geringer-Sameth et al. 2015). We omit recently published $J$ values (e.g. those of Bonnivard et al. (2015a,b); Simon et al. (2015); Zhu et al. (2016); Hayashi et al. (2016); Genina \& Fairbairn (2016); Walker et al. (2016); Bonnivard et al. (2016); Klop et al. (2017); Pace \& Strigari (2018)) since the comparison with Hydrus 1 will not be straightforward.

There have been several recent attempts to simplify the

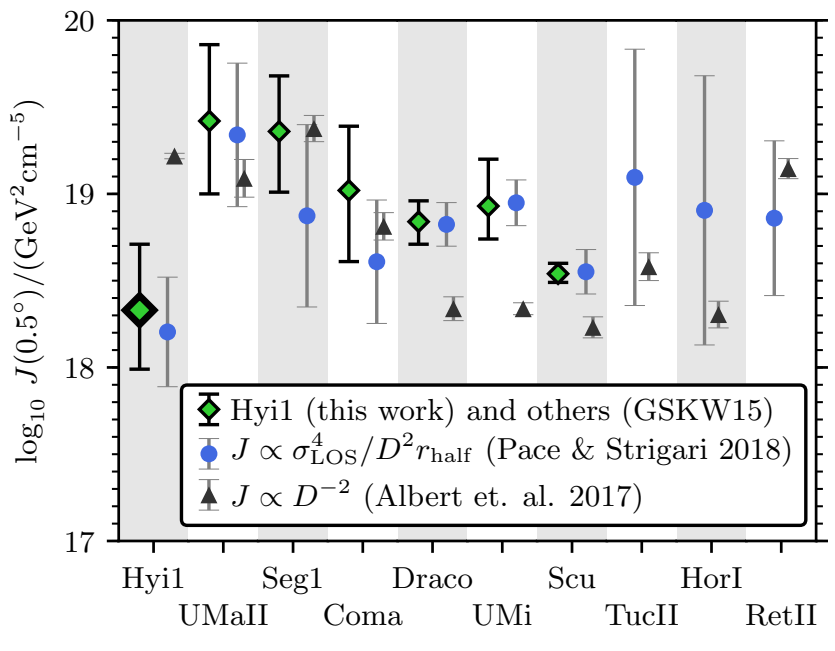

Figure 15. The astrophysical $J$ factor for dark matter annihilation of Hydrus 1 compared with those of other Milky Way dwarf spheroidals. Green diamonds show $J$ of several dwarfs determined with the procedure given in Geringer-Sameth et al. (2015) (GSKW15). Blue circles and gray triangles are estimates of $J$ based on simple scaling formulas. They are fit to the GSKW15 $J$ 's but their values for Hydrus 1 can be considered predictions.

estimation of $J$ values using functions of photometricallydetermined properties (distance, half-light radius) and, sometimes, the total line-of-sight velocity dispersion. These formulas usually have free parameters that are fit to existing compilations of $J$ values based on the Jeans equation. Hydrus 1 presents the first chance to test them. In Fig. 15 we show two predictions for the $J$ value for Hydrus 1 . Blue circles are based on the formula $J \propto \sigma_{\text {los }}^{4} D^{-2} r_{\text {half }}^{-1}$ (Pace \& Strigari 2018), whose form can be motivated by simple analytic considerations (e.g. Evans et al. 2016). In addition to calibrating to their own Jeans analysis of the dwarfs, Pace \& Strigari (2018) recalibrate the formula to the GeringerSameth et al. (2015) data set (green diamonds), finding that it fits dwarf J's with residual error of 0.08 dex. Adopting the latter calibration, we see that the scaling relation appears to fit the measured value for Hydrus 1 fairly well (error bars come from adding in quadrature the uncertainty in velocity dispersion, distance, half-light radius and a $0.08 \mathrm{dex}$ intrinsic scatter). The gray triangles in Fig. 15 correspond to a purely photometric determination of $J$, developed by Drlica-Wagner et al. (2015a); Albert et al. (2017), where $J$ is a function of distance alone, scaling as $J \propto D^{-2}$. As with the blue circles, this relation was calibrated to the results of Geringer-Sameth et al. (2015). The distance-based $J$ estimate severely overpredicts Hydrus 1's J value. While the failure of such a simple estimator is perhaps not surprising, the lesson is that spectroscopic observations of dwarf galaxies are necessary to determine their dark matter content. If the distance-based scaling is used to predict dwarf $J$ values it must be accompanied by a large error bar (Albert et al. 2017). Here, an intrinsic uncertainty $>0.8$ dex would be needed to bring the estimate to $<1 \sigma$ tension with the observationally determined $J$ of Hydrus 1 .

The relative $J$ values among different dwarfs are an essential ingredient in joint searches for an annihilation signal 


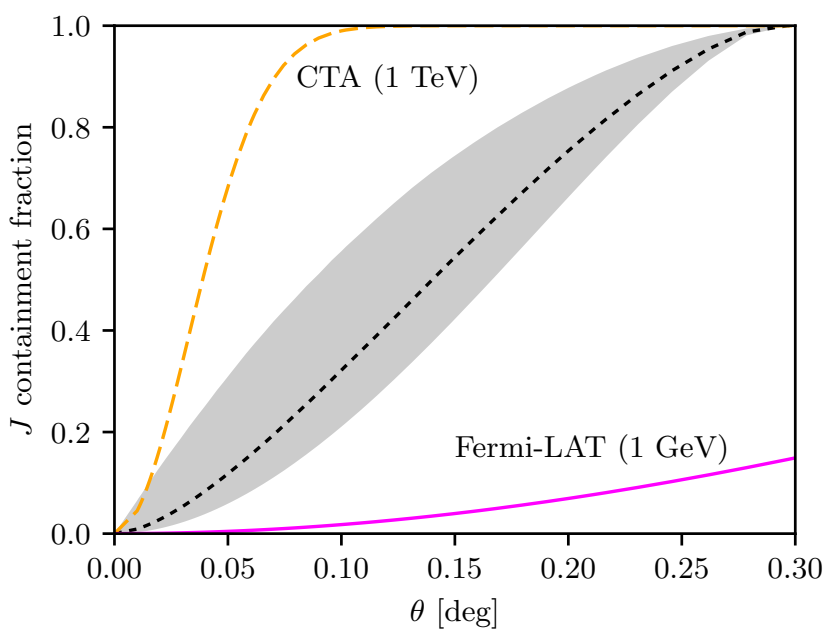

Figure 16. Constraints on the spatial extent of Hydrus 1's dark matter annihilation profile. For each angle $\theta$ away from the center of the dwarf the $J$ containment fraction gives the fraction of annihilation flux that comes from the region within $\theta$. The black dotted line is the median estimate, while the gray band shows the 16 th to 84 th percentiles of the posterior. The gamma-ray PSFs of the CTA (at $1 \mathrm{TeV}$ ) and Fermi-LAT (at $1 \mathrm{GeV}$ ) are shown for comparison. CTA will detect Hydrus 1 as an extended source.

from all dwarfs at once (e.g. Geringer-Sameth \& Koushiappas 2011; Ackermann et al. 2011; Mazziotta et al. 2012). Pointed instruments (e.g. imaging atmospheric Cherenkov telescopes) must incorporate the $J$ rankings in designing observing strategies. Ratios of $J$ 's also provide a crucial test of a dark matter origin for any signal detected from a dwarf, since fluxes must be proportional to $J$ (Strigari et al. 2007; Geringer-Sameth et al. 2015; Geringer-Sameth et al. 2015b; Albert et al. 2017). Although Hydrus 1 is not among the top tier of dwarfs in terms of expected annihilation flux it outranks most classical and ultrafaint dwarfs that are commonly used to constrain dark matter particle physics.

Hydrus 1 is close enough to earth that its dark matter annihilation may have a detectable spatial extent. A detection in gamma-rays would be a direct observation of the shape of Hydrus 1's dark matter density profile. Following Geringer-Sameth et al. (2015), Fig. 16 illustrates Hydrus 1's spatial extent. For each angle $\theta$, the shaded region shows a $68 \%$ credible interval on the fraction of annihilation flux coming from angles less than $\theta$. Approximate PSFs for Fermi-LAT and CTA are shown for comparison ${ }^{8}$. If CTA is able to detect Hydrus 1 in the future it will almost certainly be seen as an extended source. The dark matter halo truncation radius we adopt is much smaller than the scale of the Fermi-LAT PSF. If Hydrus 1's halo extends well beyond the outermost spectroscopically-observed member star it may appear extended to Fermi. However, this question is beyond the scope of our conservative analysis.

Finally, we perform a search for gamma-ray emission from Hydrus 1 using data from the Fermi Large Area Tele-

8 They are modeled as 2-d Gaussian distributions with $68 \%$ containment fractions of $0.05^{\circ}$ for CTA at $1 \mathrm{TeV}$ and $0.8^{\circ}$ for FermiLAT at $1 \mathrm{GeV}$. scope (Atwood et al. 2009). Hydrus is located $0.45^{\circ}$ in projection from a gamma-ray source 3FGL J0224.1-7941 in the Third Fermi Source Catalog (3FGL) (Acero et al. 2015), which is associated with a probable blazar. This precludes performing the search using optimized event-weighting (e.g. Geringer-Sameth et al. 2015a,b) as that method does not take into account contamination from the bright nearby source. We therefore adopt the commonly used approach based on maximum likelihood modeling of the region surrounding Hydrus 1. A best-fitting model is found both with and without a trial source at the location of Hydrus 1. If the likelihood is sufficiently improved by the addition of the trial source it is interpreted as evidence for emission from the direction of Hydrus 1.

Gamma-ray events from a $10^{\circ} \times 10^{\circ}$ region centered on Hydrus 1 are binned into $0.05^{\circ}$ spatial pixels and 25 logarithmic energy bins from $1 \mathrm{GeV}$ to $316 \mathrm{GeV}$ (10 bins per decade). The spatial binning is substantially smaller than the Fermi PSF at all energies above $1 \mathrm{GeV}$ and the analysis will be as sensitive as if we had used an unbinned likelihood function. Restricting the energy range to be greater than $1 \mathrm{GeV}$ avoids events with poor direction reconstruction and does not negatively impact the power of the search for dark matter for particle masses greater than a few $\mathrm{GeV}$ (Geringer-Sameth et al. 2015a, Sec. VI D). Our data set comprises 9.4 years of observation ${ }^{9}$. The likelihood function is the product of an independent poisson probability for each bin. The predicted number of events in a bin is the sum of isotropic ${ }^{10}$ and diffuse ${ }^{11}$ background components ${ }^{12}$ along with the four $3 \mathrm{FGL}$ sources within the $10^{\circ} \times 10^{\circ}$ region. These sources have all been associated with extragalactic objects by the Fermi Collaboration in the 3FGL and we adopt the precise locations of their counterparts rather than their coordinates in the 3FGL. Each source is modeled as a point source with a "log-parabola" energy spectrum, whose flux takes the form $d F / d E \propto E^{-(\alpha+\beta \log E)}$. The null model has 14 free parameters: normalizations of the two background components and the normalization, $\alpha$, and $\beta$ of each source. The trial point source located at the position of Hydrus 1 has an energy spectrum given by $d F / d E \propto E^{-(\alpha+\beta \log E)} \exp \left(-E / E_{c}\right)$, with 4 additional free parameters (normalization, $\alpha, \beta$, and $E_{c}$ ). This flexible energy spectrum is sufficient to describe dark matter annihilation into Standard Model leptons, quarks, and gauge bosons.

The test statistic is $\lambda_{\text {obs }}=2 \log \left(\hat{L}_{1} / \hat{L}_{0}\right)$, where $\hat{L}_{0}$ is the value of the likelihood maximized over the 14 free pa-

9 We use version v10r0p5 of the Fermi Science Tools. Front and back P8R2_SOURCE_V6 events from weeks 9 to 500 were extracted with gtselect $(z \max =90)$, and gtmktime ( ilter=" (DATA_QUAL>0) \&\& (LAT_CONFIG==1)" and roicut=no). We ran gtltcube with zmax $=90$ and gtexpcube 2 with binsz=0.1 to create an exposure map, gtpsf to compute the PSF at the location of Hydrus 1, and assume that the PSF is constant across region surrounding Hydrus 1.

10 iso_P8R2_SOURCE_V6_v06.txt

11 gll_iem_v06.fits

12 At the edges of each energy bin the background templates were multiplied by the exposure map, convolved with the PSF at a resolution of $0.05^{\circ}$ over a $25^{\circ} \times 25^{\circ}$ region, and integrated over the energy bin (assuming $\log$ flux $\propto \log$ energy) to obtain the model prediction. 
rameters of the null model without Hydrus 1 and $\hat{L}_{1}$ is the maximum likelihood value when all 18 parameters are free to vary. The detection significance ( $p$ value) is the probability that, if the null model were true, $\lambda$ would be measured to be larger than $\lambda_{\text {obs }}$. After separately maximizing over the 14 and 18 parameter model we measure $\lambda_{\text {obs }}=1.73$. Calibrating the distribution of $\lambda$ using the region surrounding Hydrus 1, we find the probability of obtaining a larger $\lambda$ under the null model is $\gtrsim 21 \%$ and conclude that the Fermi data do not show any evidence for gamma-ray emission from Hydrus 1. This is consistent with gamma-ray observations of dwarfs with larger $J$ values (e.g. Albert et al. 2017), including both non-detections as well as a potential gamma-ray signal from Reticulum II if the latter is due to dark matter annihilation (Geringer-Sameth et al. 2015b; Hooper \& Linden 2015) (see also Drlica-Wagner et al. (2015a); Albert et al. (2017)). We also search for new sources in the vicinity of Hydrus 1 by adding a trial source at each location within the $10^{\circ} \times 10^{\circ}$ region. This search reveals two probable new gamma-ray sources but they are several degrees away from Hydrus 1.

\subsection{Chemistry}

In Section 4.4 we described that we were able to get robust abundances of more than one chemical element, in particular iron, magnesium and carbon for many Hydrus members. First we look at the abundances of alpha elements vs iron elements in the satellite. The alpha element abundances are particularly useful for constraining the length of star formation history, due to the fact that Type Ia and Type II Supernovae have vastly different yields in alpha-elements and that Type Ia supernovae occur with a delay of $\sim 100 \mathrm{Myr}$ after the beginning of star formation and the first Type II supernovae explosions (Woosley \& Weaver 1995; Maoz et al. 2014). Figure 17 shows the $[\mathrm{Mg} / \mathrm{Fe}]$ vs $[\mathrm{Fe} / \mathrm{H}]$ abundance distribution for Hydrus 1 member stars, together with the distribution in the MW halo and other UFDs. We note that, on average, the $[\mathrm{Mg} / \mathrm{Fe}]$ in Hydrus's stars is larger than zero $<[\mathrm{Mg} / \mathrm{Fe}]>\sim 0.2$, which is indicative that the star-formation episode that led to the formation of Hydrus 1 was not very extended in time. However, the data also show that $[\mathrm{Mg} / \mathrm{Fe}]$ decreases significantly from $[\mathrm{Mg} / \mathrm{Fe}] \sim 0.6$ at $[\mathrm{Fe} / \mathrm{H}] \sim-3$ to almost solar at higher metallicity. This feature is associated with self-enrichment of gas by Type Ia supernovae (Tinsley 1979) and implies that the star formation proceeded for at least $\sim 100 \mathrm{Myr}$ so that the $\alpha$ abundances were diluted by Type Ia yields. The lack of higher metallicity stars with solar alpha-to-iron abundances, however, means that the star formation did not proceed longer than $100 \mathrm{Myr}-$ 1 Gyr. A similar decline in alpha abundances is very pronounced in the MW halo and MW thick disk, as well as in some classical dwarfs (Tolstoy et al. 2009) and more luminous UFDs (Vargas et al. 2013), where it tends to occur at higher metallicities. In Hydrus 1 this knee occurs at a metallicity of -2.6 .

On the bottom panel of Figure 17 we also show the metallicity distribution function (MDF) from spectral synthesis modeling of Hyi 1 stars. The distribution seems to be asymmetric with a longer tail towards high metallicities, thereby challenging our assumption of Gaussian metallicity distribution for the purposes of our chemo-dynamical

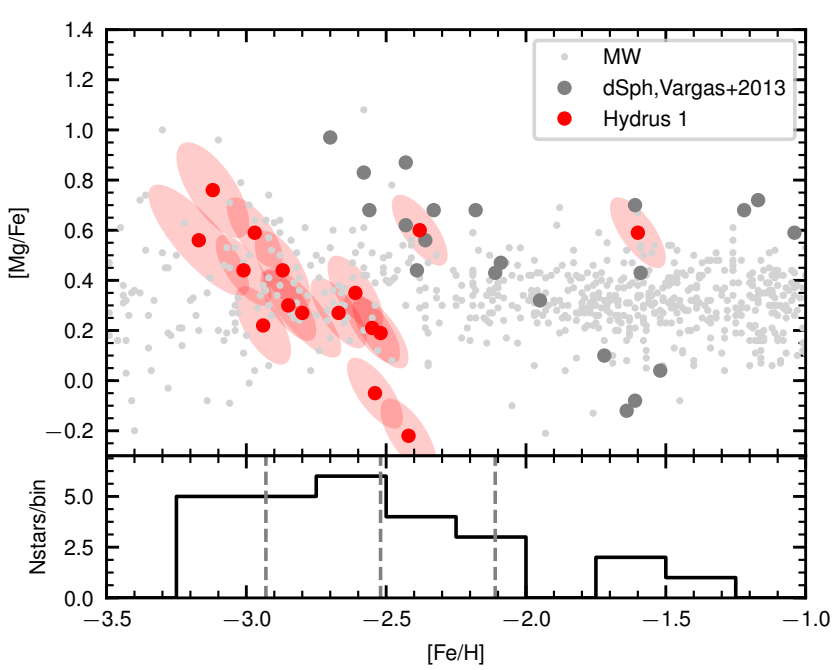

Figure 17. Chemical abundances of Hydrus 1 member stars. Top panel: $[\mathrm{Mg} / \mathrm{Fe}]$ vs $[\mathrm{Fe} / \mathrm{H}]$ for Hyi 1 stars are shown by red points with ellipses showing the 1 sigma covariance. Milky Way stars from the SAGA database (Suda et al. 2008) are shown by small light grey markers while stars from several faint dwarf spheroidals (Vargas et al. 2013) are shown by large dark grey circles. One Hydrus 1 star (star 26) is outside the plot, with $[\mathrm{Fe} / \mathrm{H}] \sim-2.15$ and $[\mathrm{Mg} / \mathrm{Fe}] \sim-0.9$ Bottom panel: Histogram of iron abundances of Hydrus 1 stars. The dashed lines show the mean and standard deviation of the Gaussian abundance distribution that was part of our chemo-dynamical model. We note that the metallicity distribution seems to be non-Gaussian, with a more pronounced metal-rich tail.

modelling. However, because of small number statistics and significant uncertainties in individual metallicity measurements, it remains to be seen whether the MDF asymmetry is real. We also remark that most dwarf galaxies show MDFs with a more pronounced metal-poor tail; however, some, such as Ursa Minor (Kirby et al. 2011), show a metal rich tail, which could be explained by chemical evolution models with lower galactic wind efficiency.

Two likely Hyi 1 member stars with higher metallicities shown in Fig. 17 have high $[\mathrm{Mg} / \mathrm{Fe}]$ ratio. The cause of this is unclear. It is possible, although unlikely, that those are MW halo contaminants rather than Hydrus 1 stars. We also remark on one star (star 26) that is outside the plotting range on Figure 17 with unusually low $[\mathrm{Mg} / \mathrm{Fe}] \sim-0.9$. Similar abundances were previously observed in some chemically peculiar stars in other dwarfs, such as Carina (Venn et al. 2012), where the unusual abundance patters were potentially associated with inhomogeneous gas mixing and star formation from SN Ia enriched gas, leading to an overabundance of iron with respect to other elements.

Upper limits on $[\mathrm{C} / \mathrm{H}]$ are available for 21 Hydrus 1 members. These limits are not informative. We can only confirm that one of the more metal-rich stars, Star 77 with $[\mathrm{Fe} / \mathrm{H}]=-1.60 \pm 0.10$, is not a carbon-enhanced metalpoor star: $[\mathrm{C} / \mathrm{Fe}]<0.54$. For all other members with upper limits on $[\mathrm{C} / \mathrm{H}]$, the limits are higher than both the $[\mathrm{C} / \mathrm{Fe}]>+0.7$ and $[\mathrm{C} / \mathrm{Fe}]>+1.0$ definitions that are commonly adopted for carbon-enhanced metal-poor stars (Aoki 
et al. 2007; Beers \& Christlieb 2005). From these limits we cannot summarise how many Hydrus 1 members meet the definition for carbon enhancement.

With $[\mathrm{C} / \mathrm{Fe}] \sim+3$ and $[\mathrm{Fe} / \mathrm{H}]=-3.18$, Star 75 clearly meets the definition of a carbon-enhanced metal-poor star. Indeed, the estimated $[\mathrm{C} / \mathrm{H}]$ ratio is near the Solar value, which is remarkable for an extremely metal-poor star in an ultra-faint dwarf galaxy. A search of the SAGA database (Suda et al. 2008) and the JINAbase (Abohalima \& Frebel 2018) reveals that Star 75 of Hydrus 1 is the most carbonenhanced metal-poor star known in a dwarf galaxy. The next most carbon-enhanced stars known in dwarf galaxies are Star 11 in Boötes I (Lai et al. 2011), and Star 7 in Segue 1 (Frebel et al. 2014), both which have $[\mathrm{C} / \mathrm{Fe}] \approx+2.3$. Similarly, in a study of 67 carbon-enhanced metal-poor stars in the Milky Way halo, only one was found to have $[\mathrm{C} / \mathrm{Fe}]>3$ (Abate et al. 2015).

Strong carbon enhancement in metal-poor stars results in depressed flux levels at bluer wavelengths (making $g$ relatively fainter than $r$ ), causing a reddening in the $g-r$ colour. This is consistent with what we observe in the second panel of Figure 5. Star 75, as marked with a cross-hair, is some $\sim 0.1$ magnitudes redder than most other members. This effect has been discussed before (Aoki et al. 2002). Since candidate members in ultra-faint dwarf galaxies are usually selected for follow-up spectroscopy based on their proximity to an isochrone (among other factors), it is likely that any similarly carbon-enhanced metal-poor stars in other ultrafaint dwarf galaxies have been overlooked. This acts to further complicate any estimates of the carbon-enhanced metalpoor star fraction among these near pristine galaxies (Frebel \& Norris 2015).

\section{CONCLUSIONS}

In this paper we presented the discovery and detailed analysis of a new ultra-faint Milky Way satellite, called Hydrus 1. Below we summarize the key findings about the system.

- The satellite is located in between the Large and the Small Magellanic Clouds on the sky at the heliocentric distance of $28 \mathrm{kpc}$ and $24 \mathrm{kpc}$ from the LMC. The luminosity of the satellite is $M_{V} \sim-4.7$ and the color-magnitude diagram indicates an old, metal-poor stellar population with several BHB stars and two RR-Lyrae. The object is mildly elliptical with an axis ratio of 0.8 , and is approximately aligned with the right ascension axis and proper motion of LMC. The circularized half-light radius of Hydrus 1 is $50 \mathrm{pc}$, and it has a surface brightness of $\sim 27.5 \mathrm{mag} / \mathrm{sq}$. arcsec, which is noticeably brighter than many typical ultra-faint dwarf spheroidals. These properties place the object in an ambiguous region between extended globular clusters and dwarf galaxies in the plane of size and luminosity.

- High-resolution spectroscopy revealed $\sim 30$ confirmed members of Hydrus 1 with mean $V_{G S R}$ of $-94 \mathrm{~km} \mathrm{~s}^{-1}$ and a well resolved stellar velocity dispersion of $2.7 \mathrm{~km} \mathrm{~s}^{-1}$. The implied mass-to-light ratio within the half-light radius is $\log _{10} M / L=1.82 \pm 0.16$, thus indicating that the system is dark matter dominated. Nevertheless, the mass-to-light ratio is uncharacteristically low in comparison to other dwarfs of similar luminosity. We speculate that it is possible that many other Galactic faint satellites with ambiguous sizes between extended clusters and dwarf galaxies also have intermediate values of mass-to-light ratios. These systems may either be globular clusters with a small amount dark matter or dwarf galaxies that lost some dark matter due to tides. Our analysis of the stellar kinematics of Hydrus 1 also reveals a tentative (at 2.5 sigma significance level) line-of-sight velocity gradient, which is compatible with a $3 \mathrm{~km} \mathrm{~s}^{-1}$ rotation of the satellite. If real, this could signify the detection of a first rotating ultra-faint dwarf galaxy.

- Based on the spatial position and the observed line-ofsight velocity, we find it highly plausible that Hydrus 1 is part of the Magellanic family of satellites. The dwarf may currently be a part of the leading debris of the LMC, having been stripped from the LMC between $0.1 \mathrm{Gyr}$ and $0.5 \mathrm{Gyr}$ ago. A connection between Hydrus 1 and the LMC should be confirmed or refuted by the incoming Gaia DR2 data.

- Due to its relative proximity to the Sun, Hydrus 1 is a very promising target for dark-matter annihilation searches, with a J-factor of $\log J=18.33 \pm 0.36$. While it is not the highest J-factor among all the MW dwarfs, it is competitive and has a small uncertainty, thanks to the high quality velocity dispersion measurement. Our analysis of the Fermi data at the location of Hydrus for the dark matter annihilation reveals no significant signal.

- Chemically, Hydrus 1 is rather metal poor, with mean $[\mathrm{Fe} / \mathrm{H}] \sim-2.5$ and significant scatter in abundances of 0.4 dex. It has an enhanced alpha-abundance $<[\mathrm{Mg} / \mathrm{Fe}]>\sim$ 0.2 and shows evidence of self-enrichment by SN Ia at timescales of $100-1000 \mathrm{Myr}$ demonstrated by the decreasing $[\mathrm{Mg} / \mathrm{Fe}]$ with increasing $[\mathrm{Fe} / \mathrm{H}]$. Analysis of $30 \mathrm{Hy}-$ drus 1 member stars revealed one carbon-enhanced extremely metal-poor star with metallicity $[\mathrm{Fe} / \mathrm{H}]<-3$ and $[\mathrm{C} / \mathrm{Fe}] \sim 3$. This level of carbon enrichment makes it one the most carbon-enhanced stars known in both the MW halo and the dwarf galaxies.

\section{ACKNOWLEDGEMENTS}

A. R. C. acknowledges support from the Australian Research Council (ARC) through Discovery Project grant DP160100637. D.M. is supported by an ARC Future Fellowship (FT160100206). D.M. and G.D.C. acknowledge support from ARC Discovery Project DP150103294. M.M. acknowledges support from NSF grant AST1312997. M.G.W. acknowledges support from NSF grant AST1412999. E.O. acknowledges support from NSF grant AST1313006. The research leading to these results has received funding from the European Research Council under the European Union's Seventh Framework Programme (FP/2007-2013) / ERC Grant Agreement n. 308024.

This project used data obtained with the Dark Energy Camera (DECam), which was constructed by the Dark Energy Survey (DES) collaboration. Funding for the DES Projects has been provided by the U.S. Department of Energy, the U.S. National Science Foundation, the Ministry of Science and Education of Spain, the Science and Technology Facilities Council of the United Kingdom, the Higher Education Funding Council for England, the National Center for Supercomputing Applications at the University of Illinois at Urbana-Champaign, the Kavli Institute of Cosmological Physics at the University of Chicago, the Center 
for Cosmology and Astro-Particle Physics at the Ohio State University, the Mitchell Institute for Fundamental Physics and Astronomy at Texas A\&M University, Financiadora de Estudos e Projetos, Fundação Carlos Chagas Filho de Amparo à Pesquisa do Estado do Rio de Janeiro, Conselho Nacional de Desenvolvimento Científico e Tecnológico and the Ministério da Ciência, Tecnologia e Inovacão, the Deutsche Forschungsgemeinschaft, and the Collaborating Institutions in the Dark Energy Survey. The Collaborating Institutions are Argonne National Laboratory, the University of California at Santa Cruz, the University of Cambridge, Centro de Investigaciones Enérgeticas, Medioambientales y Tecnológicas-Madrid, the University of Chicago, University College London, the DES-Brazil Consortium, the University of Edinburgh, the Eidgenössische Technische Hochschule (ETH) Zürich, Fermi National Accelerator Laboratory, the University of Illinois at Urbana-Champaign, the Institut de Ciències de l'Espai (IEEC/CSIC), the Institut de Física d'Altes Energies, Lawrence Berkeley National Laboratory, the Ludwig-Maximilians Universität München and the associated Excellence Cluster Universe, the University of Michigan, the National Optical Astronomy Observatory, the University of Nottingham, the Ohio State University, the OzDES Membership Consortium the University of Pennsylvania, the University of Portsmouth, SLAC National Accelerator Laboratory, Stanford University, the University of Sussex, and Texas A\&M University.

This work has made use of data from the European Space Agency (ESA) mission Gaia (https://www.cosmos . esa.int/gaia), processed by the Gaia Data Processing and Analysis Consortium (DPAC, https://www.cosmos.esa. int/web/gaia/dpac/consortium). Funding for the DPAC has been provided by national institutions, in particular the institutions participating in the Gaia Multilateral Agreement.

Based on observations at Cerro Tololo Inter-American Observatory, National Optical Astronomy Observatory (NOAO Prop. 2016A-0618 and 2017B-0906; PI: D. Mackey), which is operated by the Association of Universities for Research in Astronomy (AURA) under a cooperative agreement with the National Science Foundation.

\section{REFERENCES}

Abate, C., Pols, O. R., Izzard, R. G., \& Karakas, A. I. 2015, A\&A, $581, \mathrm{~A} 22$

Abbott, T. M. C., Abdalla, F. B., Allam, S., et al. 2018, arXiv: 1801.03181

Abohalima \& Frebel 2018, submitted (arXiv:1711.04410)

Acero F., et al., 2015, ApJS, 218, 23

Ackermann M., et al., 2011, Physical Review Letters, 107, 241302

Ackermann M., et al., 2015, Physical Review Letters, 115, 231301

Adén, D., Wilkinson, M. I., Read, J. I., et al. 2009, ApJ, 706, L150

Albert A., et al., 2017, ApJ, 834, 110

Allende Prieto, C., Sivarani, T., Beers, T. C., et al. 2008, AJ, 136, 2070

Aoki, W., Norris, J. E., Ryan, S. G., Beers, T. C., \& Ando, H. 2002, ApJ, 567, 1166

Aoki, W., Beers, T. C., Christlieb, N., et al. 2007, ApJ, 655, 492

Atwood W. B., et al., 2009, ApJ, 697, 1071

Bechtol, K., Drlica-Wagner, A., Balbinot, E., et al. 2015, ApJ, 807,50
Beers, T. C., \& Christlieb, N. 2005, ARA\&A, 43, 531

Belokurov, V., Zucker, D. B., Evans, N. W., et al. 2007, ApJ, 654, 897

Belokurov, V., Irwin, M. J., Koposov, S. E., et al. 2014, MNRAS, 441,2124

Belokurov, V., \& Koposov, S. E. 2016, MNRAS, 456, 602

Belokurov, V., Erkal, D., Deason, A. J., et al. 2017, MNRAS, 466, 4711

Belokurov, V., Deason, A. J., Koposov, S. E., et al. 2018, MNRAS,

Benson, A. J., Frenk, C. S., Lacey, C. G., Baugh, C. M., \& Cole, S. 2002, MNRAS, 333, 177

Bertin, E. 2010, Astrophysics Source Code Library, ascl:1010.068

Bertin, E. 2011, Astronomical Data Analysis Software and Systems XX, 442, 435

Bertin, E., \& Arnouts, S. 1996, A\&AS, 117, 393

Besla, G., Martínez-Delgado, D., van der Marel, R. P., et al. 2016, ApJ, 825, 20

Bonifacio, P., Caffau, E., Zaggia, S., et al. 2015, A\&A, 579, L6

Bonnivard V., et al., 2015a, MNRAS, 453, 849

Bonnivard V., et al., 2015b, ApJ, 808, L36

Bonnivard V., Maurin D., Walker M. G., 2016, MNRAS, 462, 223

Bressan, A., Marigo, P., Girardi, L., et al. 2012, MNRAS, 427, 127

Brodie, J. P., Romanowsky, A. J., Strader, J., \& Forbes, D. A. 2011, AJ, 142, 199

Brooke, J. S. A., Bernath, P. F., Western, C. M., et al. 2013, 68th International Symposium on Molecular Spectroscopy, \#ERD02

Bullock, J. S., \& Boylan-Kolchin, M. 2017, ARA\&A, 55, 343

Caldwell, N., Walker, M. G., Mateo, M., et al. 2017, ApJ, 839, 20

Carpenter, B, Gelman, A., Hoffman, M., et al. 2017, Journal of Statistical Software, 76(1)

Casey, A. R. 2014, Ph.D. Thesis,

Castelli, F., \& Kurucz, R. L. 2004, arXiv:astro-ph/0405087

Catelan, M., Pritzl, B. J., \& Smith, H. A. 2004, ApJS, 154, 633

Chabrier, G. 2003, PASP, 115, 763

Choi, J., Dotter, A., Conroy, C., et al. 2016, ApJ, 823, 102

Collins, M. L. M., Tollerud, E. J., S and, D. J., et al. 2017, MNRAS, 467, 573

The Dark Energy Survey Collaboration 2005, arXiv:astro$\mathrm{ph} / 0510346$

Deason, A. J., Belokurov, V., \& Evans, N. W. 2011, MNRAS, 416, 2903

Deason, A. J., Wetzel, A. R., Garrison-Kimmel, S., \& Belokurov, V. 2015, MNRAS, 453, 3568

Desai, S., Armstrong, R., Mohr, J. J., et al. 2012, ApJ, 757, 83

Drlica-Wagner A., et al., 2015, ApJ, 809, L4

Drlica-Wagner, A., Bechtol, K., Rykoff, E. S., et al. 2015, ApJ, 813, 109

Drlica-Wagner, A., Bechtol, K., Allam, S., et al. 2016, ApJ, 833, L5

Dotter, A. 2016, ApJS, 222, 8

Eadie, G. M., Springford, A., \& Harris, W. E. 2017, ApJ, 835, 167

Evans N. W., Sanders J. L., Geringer-Sameth A., 2016, Phys. Rev. D, 93, 103512

Fermani, F., \& Schönrich, R. 2013, MNRAS, 430, 1294

Fiorentino, G., Bono, G., Monelli, M., et al. 2015, ApJ, 798, L12

Flaugher, B., Diehl, H. T., Honscheid, K., et al. 2015, AJ, 150, 150

Foreman-Mackey, D., Hogg, D. W., Lang, D., \& Goodman, J. 2013, PASP, 125, 306

Frebel, A., Simon, J. D., \& Kirby, E. N. 2014, ApJ, 786, 74

Frebel, A., \& Norris, J. E. 2015, ARA\&A, 53, 631

Gaia Collaboration, Prusti, T., de Bruijne, J. H. J., et al. 2016, A\&A, 595, A1

Gaia Collaboration, Brown, A. G. A., Vallenari, A., et al. 2016, A\&A, 595, A2 
Gelman, A., \& Rubin, D. (1992). Inference from Iterative Simulation Using Multiple Sequences. Statistical Science, 7(4), 457-472.

Genina A., Fairbairn M., 2016, MNRAS, 463, 3630

Geringer-Sameth A., Koushiappas S. M., 2011, Phys. Rev. Lett., 107,241303

Geringer-Sameth A., Koushiappas S. M., Walker M. G., 2015a, Phys. Rev. D, 91, 083535

Geringer-Sameth A., Walker M. G., Koushiappas S. M., Koposov S. E., Belokurov V., Torrealba G., Evans N. W., 2015b, Phys. Rev. Lett., 115, 081101

Geringer-Sameth, A., Koushiappas, S. M., \& Walker, M. 2015, ApJ, 801, 74

Gibbons, S. L. J., Belokurov, V., \& Evans, N. W. 2014, MNRAS, 445,3788

Gilmore, G., Wilkinson, M. I., Wyse, R. F. G., et al. 2007, ApJ, 663,948

Goodman, J., \& Weare, J. 2010, Communications in Applied Mathematics and Computational Science, Vol. 5, No. 1, p. 65$80,2010,5,65$

Górski, K. M., Hivon, E., Banday, A. J., et al. 2005, ApJ, 622, 759

Jethwa, P., Erkal, D., \& Belokurov, V. 2016, MNRAS, 461, 2212

Jethwa, P., Erkal, D., \& Belokurov, V. 2018, MNRAS, 473, 2060

Jethwa, P., Torrealba, G., Navarrete, C., et al. 2017, arXiv:1711.09103

Hayashi K., Ichikawa K., Matsumoto S., Ibe M., Ishigaki M. N., Sugai H., 2016, MNRAS, 461, 2914

He C., Bechtol K., Hearin A. P., Hooper D., 2015, Phys. Rev. D, 91,063515

Heiter, U., Lind, K., Asplund, M., et al. 2015, Phys. Scr., 90, 054010

Henden, A. A., Levine, S. E., Terrell, D., Smith, T. C., \& Welch, D. 2012, Journal of the American Association of Variable Star Observers (JAAVSO), 40, 430

Hoffman, M. D., \& Gelman, A. 2011, arXiv:1111.4246

Hooper D., Linden T., 2015, J. Cosmology Astropart. Phys., 9, 16

Kaiser, N., Aussel, H., Burke, B. E., et al. 2002, Proc. SPIE, 4836, 154

Kaplinghat, M., \& Strigari, L. E. 2008, ApJ, 682, L93

Kazantzidis, S., Łokas, E. L., Callegari, S., Mayer, L., \& Moustakas, L. A. 2011, ApJ, 726, 98

Kirby, E. N., Guhathakurta, P., \& Sneden, C. 2008, ApJ, 682, $1217-1233$

Kirby, E. N., Cohen, J. G., Guhathakurta, P., et al. 2013, ApJ, 779,102

Klypin, A., Kravtsov, A. V., Valenzuela, O., \& Prada, F. 1999, ApJ, 522, 82

Kim, D., Jerjen, H., Milone, A. P., Mackey, D., \& Da Costa, G. S. 2015, ApJ, 803, 63

Kim, D., \& Jerjen, H. 2015, ApJ, 808, L39

Kirby, E. N., Lanfranchi, G. A., Simon, J. D., Cohen, J. G., \& Guhathakurta, P. 2011, ApJ, 727, 78

Kirby, E. N., Simon, J. D., \& Cohen, J. G. 2015, ApJ, 810, 56

Klop N., Zandanel F., Hayashi K., Ando S., 2017, Phys. Rev. D, 95, 123012

Koposov, S., \& Bartunov, O. 2006, Astronomical Data Analysis Software and Systems XV, 351, 735

Koposov, S., Belokurov, V., Evans, N. W., et al. 2008, ApJ, 686, 279-291

Koposov, S. E., Yoo, J., Rix, H.-W., et al. 2009, ApJ, 696, 2179

Koposov, S. E., Gilmore, G., Walker, M. G., et al. 2011, ApJ, 736, 146

Koposov, S. E., Belokurov, V., Torrealba, G., \& Evans, N. W. 2015, ApJ, 805, 130

Koposov, S. E., Casey, A. R., Belokurov, V., et al. 2015, ApJ, 811,62
Küpper, A. H. W., Kroupa, P., Baumgardt, H., \& Heggie, D. C. 2010, MNRAS, 401, 105

Küpper, A. H. W., Johnston, K. V., Mieske, S., Collins, M. L. M., \& Tollerud, E. J. 2017, ApJ, 834, 112

Laevens, B. P. M., Martin, N. F., Sesar, B., et al. 2014, ApJ, 786, L3

Laevens, B. P. M., Martin, N. F., Bernard, E. J., et al. 2015, ApJ, 813,44

Lai, D. K., Lee, Y. S., Bolte, M., et al. 2011, ApJ, 738, 51

Lee, Y. S., Beers, T. C., Sivarani, T., et al. 2008, AJ, 136, 2022

Lee, Y. S., Beers, T. C., Sivarani, T., et al. 2008, AJ, 136, 2050

Li S., et al., 2016, Phys. Rev. D, 93, 043518

Li, T. S., Simon, J. D., Pace, A. B., et al. 2018, arXiv:1802.06810

Mackey, A. D., Koposov, S. E., Erkal, D., et al. 2016, MNRAS, 459,239

Mackey, A. D., Koposov, S. E., Da Costa, G. S., et al. 2017, MNRAS, 472, 2975

Maoz, D., Mannucci, F., \& Nelemans, G. 2014, ARA\&A, 52, 107

Marigo, P., Girardi, L., Bressan, A., et al. 2017, ApJ, 835, 77

Martin, N. F., de Jong, J. T. A., \& Rix, H.-W. 2008, ApJ, 684, 1075-1092

Martin, N. F., \& Jin, S. 2010, ApJ, 721, 1333

Martin, N. F., Nidever, D. L., Besla, G., et al. 2015, ApJ, 804, L5

Mayer, L., Governato, F., Colpi, M., et al. 2001, ApJ, 559, 754

Mazziotta M. N., Loparco F., de Palma F., Giglietto N., 2012, Astroparticle Physics, 37, 26

McConnachie, A. W. 2012, AJ, 144, 4

McMillan, P. J. 2017, MNRAS, 465, 76

Neal, R. M. 2012, arXiv:1206.1901

Nichols, M., Colless, J., Colless, M., \& Bland-Hawthorn, J. 2011, ApJ, 742, 110

Nidever, D. L., Majewski, S. R., \& Butler Burton, W. 2008, ApJ, $679,432-459$

Pace A. B., Strigari L. E., 2018, arxiv:1802.06811

Patel, E., Besla, G., Mandel, K., \& Sohn, S. T. 2018 , arXiv: 1803.01878

Paxton, B., Bildsten, L., Dotter, A., et al. 2011, ApJS, 192, 3

Peñarrubia, J., Navarro, J. F., \& McConnachie, A. W. 2008, ApJ, 673, 226-240

Pietrukowicz, P., Kozłowski, S., Skowron, J., et al. 2015, ApJ, 811,113

Protassov R., van Dyk D. A., Connors A., Kashyap V. L., Siemiginowska A., 2002, ApJ, 571, 545

Ram, R. S., Brooke, J. S. A., Bernath, P. F., Sneden, C., \& Lucatello, S. 2014, ApJS, 211, 5

Ricotti, M., Parry, O. H., \& Gnedin, N. Y. 2016, ApJ, 831, 204

Sales, L. V., Navarro, J. F., Kallivayalil, N., \& Frenk, C. S. 2017, MNRAS, 465, 1879

Schlafly, E. F., \& Finkbeiner, D. P. 2011, ApJ, 737, 103

Schlafly, E. F., Green, G. M., Lang, D., et al. 2018, ApJS, 234, 39

Schlegel, D. J., Finkbeiner, D. P., \& Davis, M. 1998, ApJ, 500, 525

Schönrich, R., Binney, J., \& Dehnen, W. 2010, MNRAS, 403, 1829

Simon, J. D., \& Geha, M. 2007, ApJ, 670, 313

Simon J. D., et al., 2015, ApJ, 808, 95

Shanks, T., Metcalfe, N., Chehade, B., et al. 2015, MNRAS, 451, 4238

Sneden, C. A. 1973, Ph.D. Thesis,

Skrutskie, M. F., Cutri, R. M., Stiening, R., et al. 2006, AJ, 131, 1163

Smolec, R. 2005, Acta Astron., 55, 59

Strigari L. E., Koushiappas S. M., Bullock J. S., Kaplinghat M., 2007, Phys. Rev. D, 75, 083526

Suda, T., Katsuta, Y., Yamada, S., et al. 2008, PASJ, 60, 1159

Tinsley, B. M. 1979, ApJ, 229, 1046

Tollerud, E. J., Bullock, J. S., Strigari, L. E., \& Willman, B. 2008, ApJ, 688, 277-289 
Tolstoy, E., Hill, V., \& Tosi, M. 2009, ARA\&A, 47, 371

Torrealba, G., Koposov, S. E., Belokurov, V., \& Irwin, M. 2016, MNRAS, 459, 2370

Torrealba, G., Belokurov, V., Koposov, S. E., et al. 2018, MNRAS, 475,5085

Udalski, A., Szymański, M. K., \& Szymański, G. 2015, Acta Astron., 65,1

Valdes, F., Gruendl, R., \& DES Project 2014, Astronomical Data Analysis Software and Systems XXIII, 485, 379

Vargas, L. C., Geha, M., Kirby, E. N., \& Simon, J. D. 2013, ApJ, 767,134

Venn, K. A., Shetrone, M. D., Irwin, M. J., et al. 2012, ApJ, 751, 102

Voggel, K., Hilker, M., Baumgardt, H., et al. 2016, MNRAS, 460, 3384

Walker, M. G., Mateo, M., \& Olszewski, E. W. 2008, ApJ, 688, L75

Walker, M. G., Mateo, M., Olszewski, E. W., Sen, B., \& Woodroofe, M. 2009, AJ, 137, 3109

Walker, M. G., Mateo, M., Olszewski, E. W., et al. 2009, ApJ, 704,1274

Walker, M. G., Belokurov, V., Evans, N. W., et al. 2009, ApJ, 694, L144

Walker, M. G., Mateo, M., Olszewski, E. W., Bailey, III, J. I., Koposov, S. E., Belokurov, V., \& Evans, N. W. 2015, ApJ, 808,108

Walker, M. G., Mateo, M., Olszewski, E. W., Koposov, S., Belokurov, V., Jethwa, P., Nidever, D. L., Bonnivard, V., Bailey, III, J. I., Bell, E. F., \& Loebman, S. R. 2016, ApJ, 819, 53

Walsh, S. M., Willman, B., \& Jerjen, H. 2009, AJ, 137, 450

Weisz, D. R., Koposov, S. E., Dolphin, A. E., et al. 2016, ApJ, 822,32

Williams, A. A., Belokurov, V., Casey, A. R., \& Evans, N. W. 2017, MNRAS, 468, 2359

Willman, B., Dalcanton, J. J., Martinez-Delgado, D., et al. 2005, ApJ, 626, L85

Willman, B., Blanton, M. R., West, A. A., et al. 2005, AJ, 129, 2692

Willman, B., Geha, M., Strader, J., et al. 2011, AJ, 142, 128

Willman, B., \& Strader, J. 2012, AJ, 144, 76

Woosley, S. E., \& Weaver, T. A. 1995, ApJS, 101, 181

York, D. G., Adelman, J., Anderson, J. E., Jr., et al. 2000, AJ, 120,1579

Zhu L., van de Ven G., Watkins L. L., Posti L., 2016, MNRAS, 463,1117

This paper has been typeset from a $\mathrm{TEX}_{\mathrm{E}} / \mathrm{LAT}_{\mathrm{E}} \mathrm{X}$ file prepared by the author. 